\title{
Histoire humaine et naturelle d'une invasion biologique
}

L'ajonc d'Europe sur l'île de La Réunion

Human and natural history of a biological invasion: Gorse on Reunion Island

Nathalie Udo, Catherine Darrot, Michèle Tarayre et Anne Atlan

\section{OpenEdition}

\section{Journals}

Édition électronique

URL : http://journals.openedition.org/ethnoecologie/2724

DOI : $10.4000 /$ ethnoecologie. 2724

ISSN : 2267-2419

\section{Éditeur}

Laboratoire Eco-anthropologie et Ethnobiologie

\section{Référence électronique}

Nathalie Udo, Catherine Darrot, Michèle Tarayre et Anne Atlan, « Histoire humaine et naturelle d'une invasion biologique », Revue d'ethnoécologie [En ligne], 9 | 2016, mis en ligne le 01 juillet 2016, consulté le 19 avril 2019. URL : http://journals.openedition.org/ethnoecologie/2724 ; DOI : 10.4000/ ethnoecologie.2724

Ce document a été généré automatiquement le 19 avril 2019

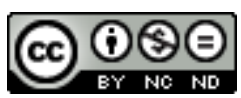

Revue d'ethnoécologie est mis à disposition selon les termes de la licence Creative Commons Attribution - Pas d'Utilisation Commerciale - Pas de Modification 4.0 International. 


\section{Histoire humaine et naturelle d'une invasion biologique}

L'ajonc d'Europe sur l'île de La Réunion

Human and natural history of a biological invasion: Gorse on Reunion Island

Nathalie Udo, Catherine Darrot, Michèle Tarayre et Anne Atlan

\section{Introduction}

1 La dynamique des espèces invasives, depuis leur introduction jusqu'à leur expansion géographique - et parfois leur régression - peut être difficile à appréhender dans son intégralité avec les méthodes classiques utilisées en écologie (comparaison de parts d'herbier, d'inventaires, d'analyses génétiques). Pourtant, connaître leurs dynamiques spatio-temporelles représente un élément central dans la compréhension globale des processus invasifs pour les biologistes et les écologues, tout comme pour les gestionnaires et les décideurs qui cherchent à prioriser et organiser leurs actions en vue de la préservation des milieux (Hierro et al. 2005, Vilà \& Ibáñez 2011). Par ailleurs, les raisons pour lesquelles certaines plantes prolifèrent et deviennent envahissantes dans les régions où elles sont introduites n'ont pas été entièrement élucidées. En particulier, peu d'études ont traité du rôle joué par le système social dans les différentes phases de la dynamique spatio-temporelle des plantes envahissantes. Pourtant, à l'ère de l'anthropocène (Bonneuil \& Fressoz 2013), la compréhension de ces processus invasifs sans la prise en compte des facteurs humains demeure limitée. Compte tenu de ces enjeux, la présente étude a un double objectif : d'une part, retracer la dynamique d'un processus invasif et, d'autre part, contribuer à élucider la part jouée respectivement - et parfois dans un « tissu sans coutures » (Hughes 1983) - par les facteurs naturels et par le système social, à l'œuvre lors des différentes étapes de l'invasion (transport, introduction, établissement, expansion spatiale, Blackburn et al. 2011). La réflexion proposée ici s'inscrit dans le courant de l'écologie historique tel que défini par Girel (2006), pour qui la biodiversité et l'humain sont liés par des interrelations complexes, et dans celui de l'écologie 
contemporaine tel que défini par Larrère \& Larrère (2012) pour qui la nature a une histoire et co-évolue avec les sociétés humaines. On pose l'hypothèse que chacune des grandes étapes du processus invasif d'une plante est profondément ancrée dans l'histoire particulière des milieux et de la société dans lesquels il s'opère, sous l'effet d'une composition inextricable d'éléments naturels et sociaux. Cette hypothèse sera explorée à la lumière de l'étude monographique de l'invasion biologique par l'ajonc d'Europe (Ulex europaeus) sur l'île de la Réunion (Océan Indien).

2 Les écologues ont abordé le sujet à travers deux questions principales : "quelles espèces peuvent devenir envahissantes? 》 et "quelles caractéristiques des écosystèmes peuvent les rendre vulnérables aux invasions?» (Pyšek \& Richardson 2006). Les études ont porté sur les caractéristiques des espèces (en particulier la croissance et la reproduction), sur les caractéristiques écologiques des milieux et les communautés d'accueil (par exemple la richesse et l'abondance spécifique), ou simultanément sur les deux aspects (Hierro et al. 2005). Une production de graines importante, une aptitude à la reproduction végétative, une forte abondance dans la zone d'origine sont des traits favorables pour qu'une espèce devienne envahissante. Parallèlement, l'absence de prédateurs de l'espèce introduite, la faible richesse spécifique et une fréquence élevée de perturbations rendent des écosystèmes plus propices à accueillir des espèces invasives. Malgré le nombre important de caractéristiques et de milieux étudiés, aucune généralisation ne s'avère possible : le succès d'une invasion dépend de l'adéquation entre l'espèce et le milieu récepteur (Facon et al. 2006), sans compter la part de hasard (Keller \& Taylor 2008). De plus, le succès de l'invasion dépend de l'histoire entre la plante et les sociétés humaines : une espèce dont l'histoire est associée à celle des sociétés a une plus grande probabilité de devenir envahissante car elle a plus de chances d'être transportée volontairement et en grande quantité et qu'elle peut être pré-adaptée aux perturbations d'origine anthropique (Suarez \& Tsutsui 2008).

Une grande partie des travaux en écologie abordent le rôle des humains dans le processus invasif au moment du transport et de l'introduction. Il est alors analysé comme un facteur qui augmente la pression de propagules, une étape considérée comme primordiale dans le succès des invasions (Colautti et al. 2006). Au cours de la phase d'expansion, de nombreuses pratiques humaines sont désignés comme des facteurs facilitateurs majeurs dans le succès de l'invasion. Ainsi, les usages du sol (agriculture intensive, urbanisation), les changements d'usage des terres (nombre, trajectoire et direction des changements), de régimes de propriétés foncières ou encore une mobilité plus importante ont été identifiés comme des facteurs explicatifs du succès des invasions à plusieurs reprises (ex : Domènech et al. 2005, Brunzel et al. 2009, Klepeis et al. 2009, Vilà \& Ibáñez 2011, Manier et al. 2014). Toutefois, rares sont les cas où ces modifications sont mises en relation avec leurs contextes socio-économiques. Pourtant, quand cela est fait, les moteurs des processus invasifs apparaissent à des échelles beaucoup plus vastes et conduisent à repenser les invasions comme des passagères de changements très globaux. L'expansion de l'ambroisie (Ambrosia artemisiifolia), par exemple, a été mise en relation avec les transitions politiques en Europe de l'Est à partir des années 1990: ces transitions ont participé à une modification de la gestion des espaces agricoles et urbains, entraînant de larges espaces perturbés propices à l'ambroisie (Chauvel et al. 2006, Kiss \& Béres 2006). À travers une approche historique de 1956 à 2003, Domènech et al. (2005) ont montré que le patron actuel de l'invasion par l'herbe de la Pampa (Cortaderia selloana) en Catalogne (Espagne) peut s'expliquer par l'héritage historique de l'utilisation des terres, entre la 
mise en place de pâtures, le nombre de modifications d'usages du sol, la subdivision des fermes, la durée d'abandon des terres et la pression d'urbanisation. À notre connaissance toutefois, l'analyse fine de la dynamique spatio-temporelle d'une espèce végétale invasive au regard des interrelations entre le contexte socio-économique et les caractéristiques biologiques et écologiques de la plante tout au long du processus invasif n'a jamais été complètement établi.

Les processus d'invasion ne peuvent donc s'appréhender dans l'ensemble qu'au travers de l'histoire complète de l'invasion, de l'introduction jusqu'à l'expansion (Chérubini 2006, Harris et al. 2007, Vilà \& Ibáñez 2011) et dans le prolongement de l'histoire de la plante dans la zone d'origine où le processus a commencé (Hierro et al. 2005). Cette compréhension globale constitue une aide importante pour la réflexion et le choix de l'action (Williams et al. 2005). Elle permettra de connaître les différentes voies d'introductions, d'identifier les facteurs favorisant l'expansion, et de connaitre les voies de dispersion. Or, ces processus temporels sont très complexes et les données incomplètes (Vilà \& Ibáñez 2011) : il y a souvent des introductions multiples, volontaires ou involontaires ; l'histoire du milieu récepteur peut évoluer au fil du temps, etc. De plus, les espèces introduites, avant de devenir envahissantes, connaissent une phase de latence qui peut s'étendre de quelques années à plus de cent ans (Mack et al. 2000), ce qui renforce l'idée que chaque histoire est particulière. Les méthodes classiques mobilisées pour retracer l'histoire des invasions (comparaison de parts d'herbier, d'inventaires, de cartographies, d'analyses génétiques) ne sont pas toujours applicables : soit que l'échelle de temps soit incompatible avec la résolution temporelle de ces techniques, soit que les cartographies ou les parts d'herbiers soient trop parcellaires ou hétérogènes, soit que l'espèce présente une structure génétique inadaptée aux analyses phylogéographiques. Nous proposons dans cet article une nouvelle approche méthodologique, transdisciplinaire, pour atteindre notre double objectif, la mise en évidence conjointe de la dynamique de l'ajonc et des facteurs explicatifs socio-naturels des différentes étapes du processus.

\section{Matériels et méthodes}

\section{Approche méthodologique}

5 Notre double objectif nécessite une approche méthodologique transdisciplinaire (Piajet 1967) et par là même hybride, une approche «bricolée » pour une meilleure élucidation du réel (Odin \& Thuderoz 2010). En effet, des données de nature très variées (sociétales, écologiques, géographiques), souvent parcellaires et à des échelles spatiales variables, doivent être collectées et ce sur une large période historique (plusieurs centaine d'années). Dès lors, les cartes de répartitions côtoient les archives du XIX ${ }^{e}$ siècle disponibles; les entretiens semi-directifs joignent des observations de terrains et des inventaires floristiques sont mis en relation avec des études anthropologiques et sociologiques. Ces différents types de données, collectés en simultané au fil d'aller-retour constants et analysés par recoupements méthodiques par espace et par époque, contribuent à constituer

« un ensemble à chaque instant fini d'outils et de matériaux, [...] contingent de toutes les occasions qui se sont présentées de renouveler ou d'enrichir le stock [...] en vertu du principe que ça peut toujours servir » (Lévi-Strauss 1962). 
6 L'ajonc, tout comme l'île de La Réunion, possèdent les caractéristiques nécessaires à la mise en application d'une telle approche théorique et méthodologique.

\section{L'ajonc d'Europe}

\section{Une espèce historiquement liée à l'humain}

7 L'ajonc est une espèce très liée à l'histoire des sociétés humaines. Dans sa zone d'origine, les pays celtiques d'Europe de l'Ouest, il était utilisé comme auxiliaire agricole multiusages (clôture, fourrage, engrais vert, litière). Dans les régions où il était cultivé, notamment en Bretagne, il avait une grande importance économique et culturelle au XIX siècle (Beaulieu \& Pouëdras 2014). Durant la constitution des grands empires coloniaux européens, il a été introduit volontairement, essentiellement pour ses usages de fourrage et de clôture, mais aussi pour des raisons affectives, sur tous les continents et dans plus d'une vingtaine d'îles océaniques, dont l'île de La Réunion (Kueffer et al. 2010, Atlan et al. 2015). Dans la majorité des régions où il a été introduit, il est aujourd'hui considéré comme une espèce invasive majeure (Holm et al. 1997, Hill et al. 2008).

\section{Une espèce pionnière}

8 L'ajonc est un buisson épineux pouvant vivre jusqu'à 30 ans. À partir de 2 à 3 ans, l'ajonc fleurit plusieurs mois par an et la production annuelle de graines -bien que très variablepeut atteindre plus de 10000 graines par individu et par an. Ces graines conservent leur capacité germinative pendant plusieurs années, voire dizaines d'années (Rees \& Hill 2001). L'ajonc affectionne essentiellement les milieux ouverts (landes et friches) dans lesquels il peut former des fourrés denses et impénétrables (Richardson \& Hill 1998). De plus, l'ajonc est une espèce pyrophile : sa présence favorise les incendies en raison de son fort pouvoir de combustion (Hely \& Forgeard 1998) et le passage du feu déclenche la germination des graines (Zabkiewicz \& Gaskin 1978). Toutes ces caractéristiques biologiques font de l'ajonc une espèce qui peut rapidement coloniser des espaces perturbés. Dans la zone d'origine, les pratiques culturales tels que les incendies volontaires, les fauches, les défrichements ont ainsi joué un rôle important dans l'évolution des landes et la dynamique de l'ajonc (Bourlet 1980).

\section{Une espèce facilement reconnaissable}

9 Les ajoncs sont en fleurs presque toute l'année (Tarayre et al. 2007) ; leurs fleurs jaunes sont visibles de loin et se remarquent nettement dans le paysage réunionnais d'altitude dont la végétation indigène est majoritairement composée de plantes sans fleurs ou à petites fleurs discrètes. Du fait de sa morphologie particulière (plante très épineuse) et de l'odeur caractéristique de ses fleurs (coco) les confusions avec d'autres espèces exotiques ou indigènes sont très peu probables. De plus, l'ajonc étant historiquement un auxiliaire agricole et une plante emblématique dans sa région d'origine (Beaulieu \& Pouëdras 2014) sa reconnaissance par les explorateurs, botanistes, et agronomes en provenance d'Europe est quasi-certaine. 


\section{Lîle de La Réunion}

10 La Réunion est une île tropicale française dans le sud-ouest de l'océan Indien. Ile volcanique, montagneuse (point culminant à $3069 \mathrm{~m}$ d'altitude), au relief accidenté, elle comporte sur une petite superficie $\left(2512 \mathrm{~km}^{2}\right)$ une multitude de climats et d'habitats différents (Strasberg et al. 2005). Les pentes sont sillonnées par de nombreux cours d'eau encaissés, allant des sommets jusqu'à la mer, formant des « ravines » pouvant atteindre plusieurs dizaines de mètres de largeur et de profondeur.

11 Traditionnellement, deux grands ensembles sont distingués à la Réunion. Les Bas (< 400-600 m d'altitude) sont davantage cultivés, notamment en canne à sucre, sur des pentes à peu près régulières et concentrent également les zones urbaines. Les Hauts (> $600 \mathrm{~m}$ d'altitude) sont moins anthropisés et plus sauvages d'aspect, présentent des reliefs plus importants, sont plus boisés et accueillent les zones d'élevage. Dans les Hauts du nord-ouest, trois cirques sculptent l'île. Le cirque de Mafate est le seul concerné par l'ajonc d'Europe. Il n'est accessible qu'à pied. Il est dominé par le Massif du Maïdo. Ces deux régions sont séparées d'un rempart quasi-vertical de plus de $1000 \mathrm{~m}$ de hauteur. Dans les Hauts du sud-est s'étend le Massif du Volcan. Ces espaces naturels des Hauts de l'île ont été classés en 2007 en Parc National ( $40 \%$ de la superficie de l'île) et inscrits au patrimoine mondial de l'Unesco en 2012. Entre les trois cirques et le Massif du Volcan se trouvent deux plaines d'altitudes, aux pentes plus douces et au climat tempéré. La plus grande, la Plaine des Cafres, est une zone aujourd'hui majoritairement consacrée à l'élevage bovin. L'ajonc s'étend dans les Hauts (1000 à 2500 mètres d'altitude), à la fois dans des paysages agricoles et dans des paysages naturels, au sein de la végétation altimontaine reconnue comme l'une des plus riches en espèces endémiques de l'île. La Plaine des Cafres et le Massif du Maïdo constituent les sites d'observation privilégiés dans cette étude.

12 La Réunion a été découverte dès le $\mathrm{xV}^{\mathrm{e}}$ siècle mais elle n'a été habitée de façon pérenne qu'à partir de 1663 par des colons français et des malgaches. À partir du xvIII ${ }^{e}$ siècle, de nombreux esclaves sont débarqués sur l'île pour satisfaire le besoin de main d'œuvre particulièrement dans les plantations de café, puis, plus tard, dans les plantations de canne à sucre dans les Bas de l'île. Dès le XVII ${ }^{\mathrm{e}}$ siècle, des esclaves ont fui les plantations pour se réfugier et s'installer dans les Hauts de l'île, en particulier dans les cirques car leur difficulté d'accès offrait une protection relative aux fuyards, souvent activement traqués. L'esclavage est aboli en 1848. Aux côtés des esclaves, les colons européens sont répartis en deux catégories, d'une part "les gros blancs", les grands propriétaires terriens et, d'autre part, les " petits blancs des Hauts » dont le statut social est peu élevé. Ces derniers ont aussi cultivé les terres des Hauts (cirques et plaines d'altitude). La paupérisation d'une partie des colons européens distingue La Réunion de nombreuses autres colonies. L'̂̂le de La Réunion devient un département français en 1946. Elle est aujourd'hui soumise aux législations et administrations françaises et européennes.

13 L'île de La Réunion, de par ces caractéristiques historiques, géographiques et politiques, constitue un laboratoire idéal pour une étude d'écologie historique fondée sur un jeu de données hybrides : (i) elle est habitée de façon pérenne depuis 350 ans seulement, et l'a quasi toujours été par une seule nation, (ii) la transformation des paysages par les humains est récente et bien documentée, (iii) des archives sont disponibles sur la quasiintégralité de l'histoire de colonisation, (iv) sa taille réduite permet d'appréhender les 
phénomènes naturels et sociaux dans leur ensemble et (v) elle concentre sur un espace restreint un jeu d'acteurs sur la gestion des milieux naturels, caractérisé par une superposition d'institutions et de législations aux échelles locale, nationale et européenne (Atlan \& Darrot 2012).

\section{Collecte des données}

\section{Cartographie de la répartition actuelle}

Un élément essentiel afin de pouvoir décrire la dynamique spatio-temporelle de progression des populations d'ajoncs est d'établir la carte de répartition actuelle de l'ajonc sur l'île. Cette carte a été effectuée en compilant plusieurs bases de données géoréférencées établies par l'Office National des Forêts (ONF), le Parc National de La Réunion (Massif du Maïdo, contours des zones d'ajonc, Payet 2012 et Massif du Volcan, maille de 1 $\mathrm{km}^{2}$, Beltran 2011) et le Conservatoire Botanique National de Mascarin (base de données Cadetiana) (CBNM 2016). Ces organismes prospectent régulièrement les milieux naturels protégés et actualisent en permanence leurs bases de données, ce qui nous amène à considérer la cartographie actuelle de l'ajonc dans ces milieux comme exhaustive. Les contours des populations d'ajoncs ont été affinés par une prospection sur le terrain. Dans les zones non prospectées par ces organismes (i.e. les zones non protégées), la carte a été complétée en effectuant des prospections de terrain. Dans les plaines agricoles d'altitude, une prospection par maille de $200 \times 200 \mathrm{~m}$ le long des routes et des sentiers a été effectuée. Bien que toutes les mailles de cette zone n'aient pas été prospectées (certaines étaient situées dans des propriétés privées), le contour de la zone avec ajoncs a pu être précisément délimité. Dans le reste de l'île la présence éventuelle, passée ou présente, de l'ajonc a été documentée par les experts botanistes de La Réunion mentionnés ci-dessus et par les experts agricoles. Du fait de la diversité des méthodes de collectes il a été décidé de représenter uniquement le contour des zones avec ajoncs pour les foyers étendus et de signaler par des points la localisation des petits foyers et des pieds isolés.

\section{Sources documentaires}

Les documents historiques consultés ont contribue à décrire et expliquer la dynamique de l'ajonc sur l'île de La Réunion. Nous avons consulté des documents bibliographiques et autobiographiques des botanistes, jardiniers et explorateurs du XVII ${ }^{\mathrm{e}}$ au XIX ${ }^{\mathrm{e}}$ siècle (plus de 20 documents) dans lesquels nous avons cherché les traces des premières descriptions de l'ajonc. Nous avons parcouru des documents de littérature académique et grise (revues et ouvrages en géographie, botanique, agronomie, agriculture, ingénierie et aménagement du territoire) du xix ${ }^{e}$ siècle à aujourd'hui (plus de 500 documents), à travers lesquels nous avons cherché à la fois des mentions de l'ajonc et des éléments de contextes socio-économiques ayant agi en synergie avec les caractéristiques biologiques de l'ajonc en faveur de son expansion géographique. Pour les mêmes raisons, nous avons étudié de nombreux dossiers des archives disponibles (archives départementales de Saint-Denis, archives des évêchés, archives de l'Association Développement Rural Réunion). Enfin, les fiches d'inventaires de Zones Naturelles d'Intérêt Écologique Faunistique et Floristique (ZNIEFF) réalisées de 1980 à 2000, décrivant le milieu et les espèces végétales, nous ont permis d'identifier des zones où l'ajonc a été présent mais ne l'est plus aujourd'hui et de mettre en relation l'expansion de l'ajonc avec des 
perturbations du milieu (255 fiches). Parmi les documents consultés, 141 documents mentionnent directement l'ajonc (6 avant 1900, 12 entre 1900 et 1949, 99 entre 1950 et 2010, 24 entre 2011 et 2014).

\section{Entretiens semi-directifs}

Des entretiens semi-directifs ont été conduits auprès des usagers des espaces abritant les principales populations actuelles d'ajonc mis en évidence par la carte de répartition. Tout comme pour les documents, les principaux thèmes abordés dans les entretiens visent à apporter des éléments pour les deux objectifs. Ils concernent l'introduction de l'ajonc sur l'île, la dynamique paysagère du lieu de vie et/ou de travail de l'enquêté de son enfance à aujourd'hui, le contexte social, économique et technique. Les individus enquêtés ont été choisis en fonction de leur expérience en lien avec des végétaux sur toute ou partie de La Réunion et sur une large gamme d'âges (de 28 à 94 ans). Au total, 48 entretiens ont été réalisés entre 2013 et 2014: 15 éleveurs, 15 agents de l'ONF ou du Parc National, 7 habitants et usagers, 5 botanistes, 6 experts du territoire et de l'aménagement rural. Parmi les enquêtés, il y avait 45 hommes et 3 femmes. Ce déséquilibre est important mais reflète la situation dans le monde agricole et forestier (Insee 2008).

L'ajonc fait l'objet d'un nombre important d'autres dénominations à La Réunion. Il peut être appelé ajonc (épineux), ajonc marin, genêt (épineux), z'épine, z'épinard (des Hauts), épinard, sapan. Tout ce vocabulaire a été pris en compte dans les recherches documentaires comme dans les entretiens. Dans la suite du document nous avons employé le terme ajonc, en dehors des citations d'enquêtés où nous préservons le vocabulaire original.

\section{Résultats}

Les résultats sont structurés en quatre grandes parties : la répartition actuelle de l'ajonc à La Réunion, le contexte des premières introductions sur l'île, l'histoire des ajoncs dans l'espace agricole de la Plaine des Cafres, puis celle dans le Massif du Maïdo. Dans chacun de ces espaces sont présentés successivement la dynamique spatiale de l'ajonc et les facteurs naturels et sociétaux explicatifs.

\section{Répartition actuelle}

L'ajonc est présent dans trois foyers principaux et dans des petits foyers isolés à leur périphérie (Figure 1). Premièrement, sur la zone agricole de la Plaine des Cafres, il est présent sur une surface de l'ordre de $80 \mathrm{~km}^{2}$ et forme des fourrés denses continus sur une surface de l'ordre de $10 \mathrm{~km}^{2}$. Deuxièmement, l'ajonc est présent dans la zone hybride milieu naturel/agricole nommée Piton de l'eau (incluse dans le Massif du Volcan) sur une surface de l'ordre de $15 \mathrm{~km}^{2}$ et forme des fourrés denses sur une surface de $5 \mathrm{~km}^{2}$. Ces fourrés denses se concentrent principalement au sein et aux abords des concessions agricoles (enclaves cultivées louées par l'ONF à des éleveurs de bovins en pâturage extensif). Troisièmement, dans le Massif du Maïdo, il est présent sur une surface de l'ordre $14 \mathrm{~km}^{2}$ et forme des fourrés denses continus sur une surface de $2,5 \mathrm{~km}^{2}$. Les foyers en milieu naturel protégé se sont avérés nettement plus petits que celui de la zone 
agricole de la Plaine des Cafres qui avait jusqu'à présent échappé aux efforts de cartographie des chercheurs et gestionnaires.

Figure 1 : Répartition actuelle de l'ajonc d'Europe (Ulex europaeus) sur l'île de La Réunion en fonction de la densité des foyers (2014)

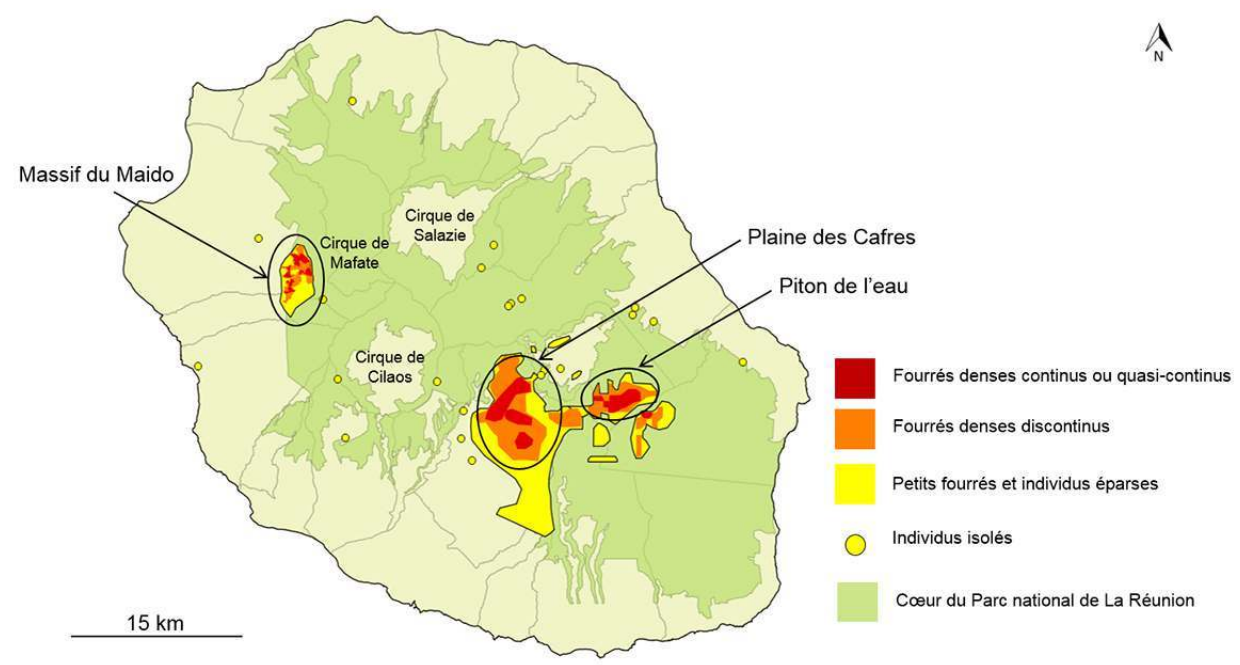

\section{Une présence sur l'île vieille de plus de 190 ans} naturalisation de Saint-Denis. Le botaniste-jardinier qui tient un catalogue des espèces cultivées précise que l'ajonc est originaire de France. Toutes les espèces originaires de France plantées dans ce jardin venaient sous forme de graines du Muséum National d'Histoire Naturelle de Paris, ce qui est donc sans doute le cas de l'ajonc. Il a été introduit à La Réunion pour un " usage économique » (Bréon 1825), ce qui, au XIXe, était souvent synonyme d'usage agricole, car l'agriculture était la base économique de l'île (Cadet 1977). En 1856, l'ajonc est de nouveau cité dans le Catalogue des végétaux cultivés au jardin du Gouvernement à l'île de La Réunion (Richard 1856). En 1853, il a été présenté à l'exposition agricole et industrielle de Saint-Denis (Moreau [1858] 1975). Ainsi, il semble que l'ajonc ait été cultivé au jardin de Saint-Denis au moins pendant 30 ans, en vue de sa naturalisation pour un usage économique agricole. À l'altitude du jardin ( $578 \mathrm{~m})$ et sous les conditions climatiques en résultant, la naturalisation de l'ajonc n'est pas possible (Hornoy 2012).

21 L'ajonc est présent sur l'île depuis plus de 190 ans, ce qui représente plus de la moitié de la période d'occupation de lîle par les humains (350 ans). Dans les deux parties suivantes, nous allons voir pourquoi et comment l'ajonc a été introduit et s'est installé et étendu dans deux des foyers actuels principaux, d'une part dans un milieu agricole, la Plaine des Cafres, et d'autre part, dans un milieu naturel, le Massif du Maïdo.

\section{L'ajonc et les agriculteurs de la Plaine des Cafres}

La plus ancienne description de l'ajonc naturalisé concerne la Plaine des Cafres; elle remonte à 1862 par Camille Jacob de Cordemoy (Jacob de Cordemoy [1862] 2004). Les textes plus anciens (de 1800 à 1850), écrits par des botanistes, voyageurs ou ingénieurs

Revue d'ethnoécologie, 9 | 2016 
européens listent des plantes européennes (avec un certain patriotisme) (Bory de SaintVincent 1804, Textor De Ravisi 1850) mais l'ajonc n'est jamais mentionné. À partir de 1862, l'ajonc est décrit de manière récurrente sur la plaine, jusqu'à aujourd'hui.

\section{Phase d'introductions de l'ajonc à La Plaine des Cafres}

\section{Une localisation et une datation précises dans les mémoires}

Plusieurs enquêtés de la Plaine des Cafres, habitants et éleveurs, ont donné des informations concordantes sur une localisation précise du lieu d'introduction de l'ajonc, dans une Ferme Modèle créée en 1849 par le célèbre Bois Joly Potier ${ }^{1}$ et exploitée pour l'élevage jusqu'en 1889 (propriété de 382 ha) (Bois Joli Potier 1871). Un couple d'éleveurs racontent

«nos parents disaient que c'était il y a longtemps. Un ancien régisseur des esclaves, Bois Joly Potier, c'est lui qui aurait introduit l'ajonc», et un habitant «moi, j'ai toujours entendu que c'était Bois Joly Potier qui a introduit les zépines à la Plaine des Cafres, dans la ferme modèle » (2013).

Actuellement, cette zone est exploitée par une coopérative agricole dont les terrains sont très envahis par l'ajonc. Les écrits confirment que dans sa volonté de coloniser la plaine, de lancer et de développer la culture et l'élevage, Bois Joly Potier a introduit plusieurs espèces exotiques, bien que l'ajonc ne soit pas explicitement mentionné (Mahy 1891, Patu de Rosemont 1856, Lavaux 1998). Il est donc très probable que l'ajonc ait été planté pour la première fois autour de 1850 sur la Ferme Modèle et il est certain qu'il s'est naturalisé peu de temps avant 1862. Dans l'encyclopédie de La Réunion (Chaudenson 1980, tome 3, la vie rurale), l'introduction de l'ajonc est imputée à Lépervanche, sous-inspecteur des Forêts et propriétaire de terrain sur la Plaine; elle est datée en 1883. Il s'agirait d'une seconde introduction sur la plaine. L'ajonc aurait donc été introduit au XIX dans au moins deux fermes de la Plaine des Cafres (Figure 2a). 
Figure 2 : Dynamique d'expansion de l'ajonc d'Europe à la Plaine des Cafres $(a, b, c)$ et sur le Massif du Maïdo (d, e, f)

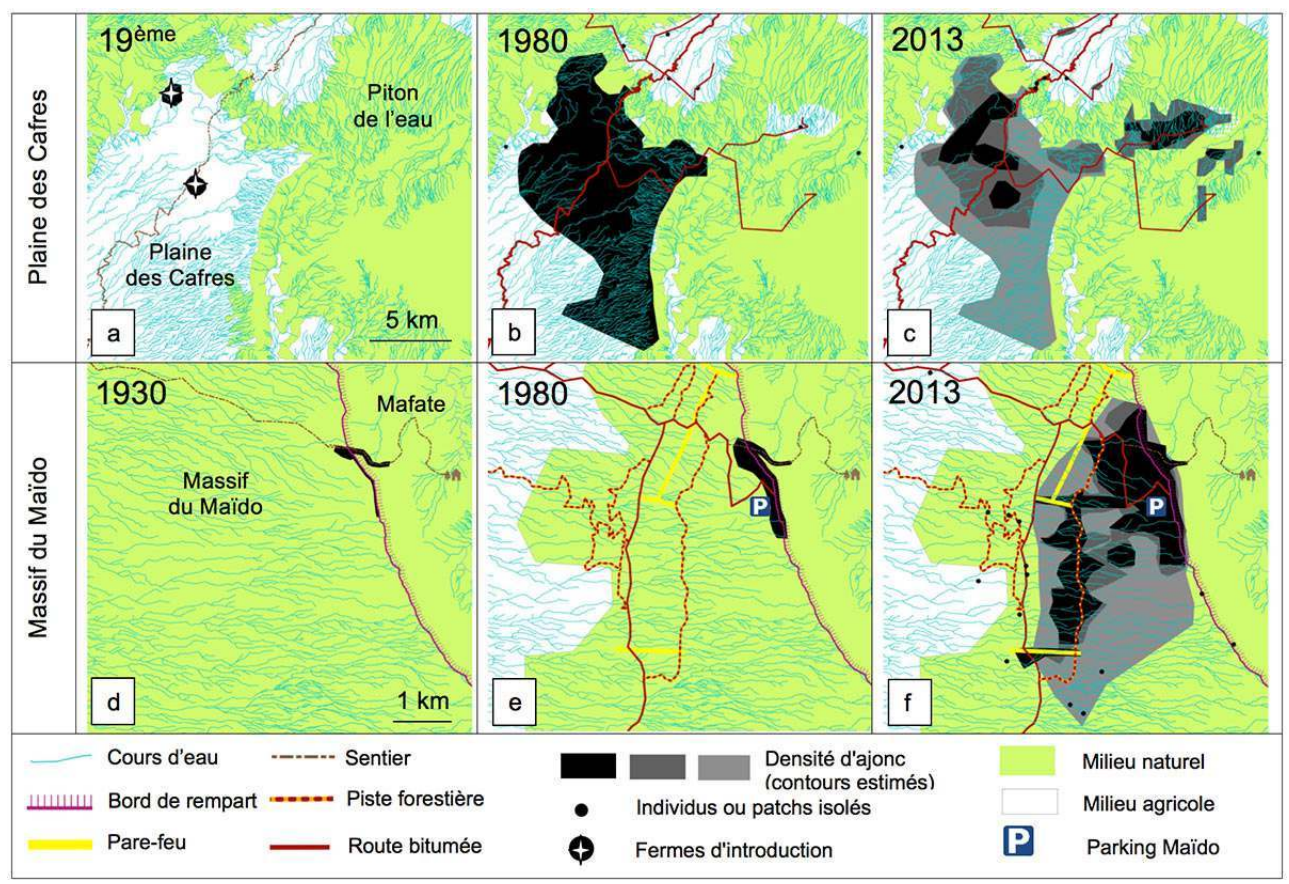

\section{Des motivations utiles et esthétiques de l'introduction} l'ajonc son usage dans l'alimentation animale (ânes, moutons, chèvres, chevaux, bovins). Cet usage est mentionné par les éleveurs racontant l'introduction de l'ajonc à la «Ferme Modèle » tenue par Bois Joly Potier. Un ethnobotaniste (Lavergne 1978, 1980) mentionne une introduction de l'ajonc à la Plaine des Cafres au cours du XIX ${ }^{\mathrm{e}}$ comme fourrage, usage qui aurait notamment été testé par Lépervanche. Un article de 1937 portant sur l'élevage rationnel dans l'île de La Réunion (Hanens 1937) indique que «l'ajonc joue, dans l'alimentation du bétail à La Réunion, un rôle de premier plan », notamment par le fait qu'il est le seul fourrage disponible toute l'année, mais l'auteur ne précise pas si c'est là la raison d'introduction. Cependant, aucun enquêté ne mentionne ni l'introduction ni l'existence à La Réunion de broyeurs utilisés en France et indispensable à la préparation de l'ajonc ; et 
seul l'article d'Hanens (1937) fait état de "broyeur » et d' "ajonc broyé » sur l'île, mais " préparés sans soin et distribué irrégulièrement ». À La Réunion, l'ajonc a davantage été utilisé comme aliment sans préparation, pour les moutons et les chèvres qui peuvent s'en nourrir sur pied et non pas comme fourrage pour les bovins à l'étable. Deux hypothèses ont été formulées pour expliquer le non transfert des savoir-faire de préparation fourragère de l'ajonc de France vers La Réunion: d'une part l'inadéquation entre préparation fourragère et type d'agriculture pratiqué à La Réunion (très extensive, en parcours), et d'autre part, l'absence de transferts des savoir-faire de la France métropolitaine vers La Réunion (Atlan et al. 2015).

\section{Les clôtures défensives}

Une autre motivation d'introduction de l'ajonc au XIX ${ }^{\mathrm{e}}$ fréquemment mentionnée par les enquêtés et citée dans l'Atlas linguistique et ethnographique de la Réunion de Carayol \& Chaudenson (1984) est l'utilisation de l'ajonc afin de constituer des haies vives ou clôtures défensives, "pour empêcher les gens de rentrer dans la ferme, [...] dans les champs ", ou " pour empêcher les animaux de sortir et pâturer dans les espaces communs ». La constitution de clôtures répond au contexte règlementaire du milieu du XIX ${ }^{e}$ siècle. En effet en 1851, lorsque les concessions de la Plaine des Cafres sont attribuées, les bénéficiaires devaient obligatoirement les enclore «soit par des haies ou tout autre moyen de défense dans les quatre ans, à compter du jour de la mise en possession, et par quart d'année en année ", sous peine de se voir confisquer « toutes les parties qui ne seraient pas closes » (Textor De Ravisi 1852). Or l'île de La Réunion ne compte aucune plante indigène ou endémique épineuse pouvant constituer des clôtures défensives (com. pers. Joël Dupont, Société Réunionnaise pour l'Étude et la Protection de la Nature). L'ajonc pouvait remplir efficacement ce rôle, qu'il remplissait par ailleurs bien en Europe et dans de nombreuses autres colonies européennes (Atlan et al. 2015). De nombreux champs sont encore bordés d'ajoncs aujourd'hui (Figure 3). 


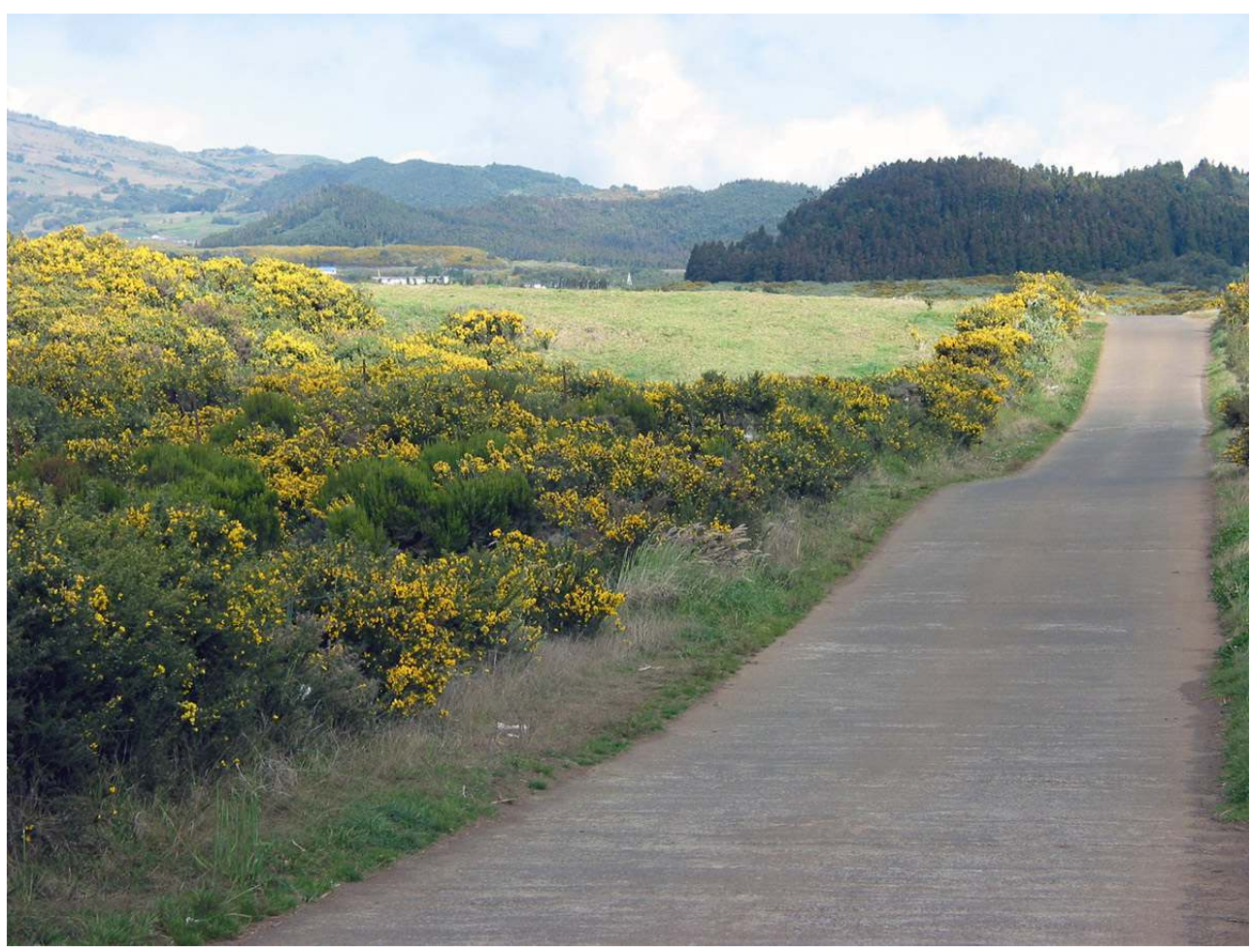

Cliché : N. Udo, 2014

\section{La nostalgie}

À l'image de la légende locale, des auteurs lient l'introduction de l'ajonc à des motivations de l'ordre de l'esthétique, l'affectif et la nostalgie. Il s'agit toujours d'ouvrages rédigés bien après que l'introduction ait effectivement eu lieu. L'introduction est alors toujours liée à la Bretagne et imputée tantôt à un religieux, «introduit, dit-on, par un prêtre breton soucieux d'évoquer sa lande natale " (Benda 1956), tantôt à des colons : " un colon breton qui avait la nostalgie de la lande natale fit venir quelques plants d'ajonc qu'il planta devant sa porte " (Vailland 1964, Lavergne 1980, Bonniol \& Benoist 1994). Certains enquêtés font également état de cette introduction sentimentale. Une introduction par les bretons est vraisemblable; ils sont en effet nombreux à être venus s'installer à La Réunion et notamment à la Plaine des Cafres (Bourde de la Rogerie 1998). De plus, l'ajonc est une plante très emblématique en Bretagne au XIX ${ }^{e}$ siècle. En revanche, une introduction initiale par un curé breton semble très peu probable: la paroisse la plus proche de La Plaine des Cafres a ouvert en 1859 et se situe à plus de $25 \mathrm{~km}$. Le premier curé breton qui y a séjourné est arrivé en 1864, soit après que l'ajonc soit naturalisé. Ce curé n'a donc pas pu être à l'origine de la première introduction.

Les motivations liées à l'agriculture et à l'affectif ne s'opposent pas, bien au contraire. Certains enquêtés mentionnent bien les deux aspects et finissent presque toujours par dire que l'ajonc a été « introduit parce qu'ils trouvaient ça joli tout compte fait » (un éleveur, 2013). 


\section{Dynamique spatio-temporelle à la Plaine des Cafres}

\section{Une longue phase d'expansion suivie d'une récente phase de régression}

Une lecture chronologique des descriptions de l'ajonc dans son milieu permet de mettre en évidence l'évolution d'un ajonc "aux allures de plante indigène " (Jacob de Cordemoy 1895) vers une espèce dominante dans les milieux ouverts, jusqu'à s'étendre dans les années 1970 sur 7000 ha (Université de la Réunion 1973) (Tableau 1 et Figure 2b). Ainsi, en à peine plus de 100 ans, il s'est installé sur toute la Plaine des Cafres de 1000 à $1700 \mathrm{~m}$ d'altitude environ'2 ${ }^{2}$ À partir de 1980-1990, la répartition de l'ajonc est beaucoup moins décrite dans les documents concernant la Plaine des Cafres, hormis dans les itinéraires techniques mis en place pour le supprimer ou le contrôler (ONF 1987, Blanfort 1996, Barbet-Massin et al. 2003), ce qui nous informe sur le fait qu'il était toujours présent et abondant, mais géré. Ainsi, ce sont davantage les entretiens auprès des éleveurs et des responsables agricoles (Association Réunionnaise de Pastoralisme ARP, Chambre d'agriculture CA974, Groupement de Défense Sanitaire GDS, ONF) qui ont apporté des informations sur la dynamique de l'ajonc pendant les 35 dernières années. La quasitotalité de ces enquêtés s'accordent sur le fait qu'il y a moins d'ajoncs aujourd'hui que dans le passé et que cette diminution des «landes à ajoncs » a commencé dans les années 1980-1990.

Tableau 1 : Les différentes étapes de la dynamique de l'ajonc d'Europe à La Plaine des Cafres

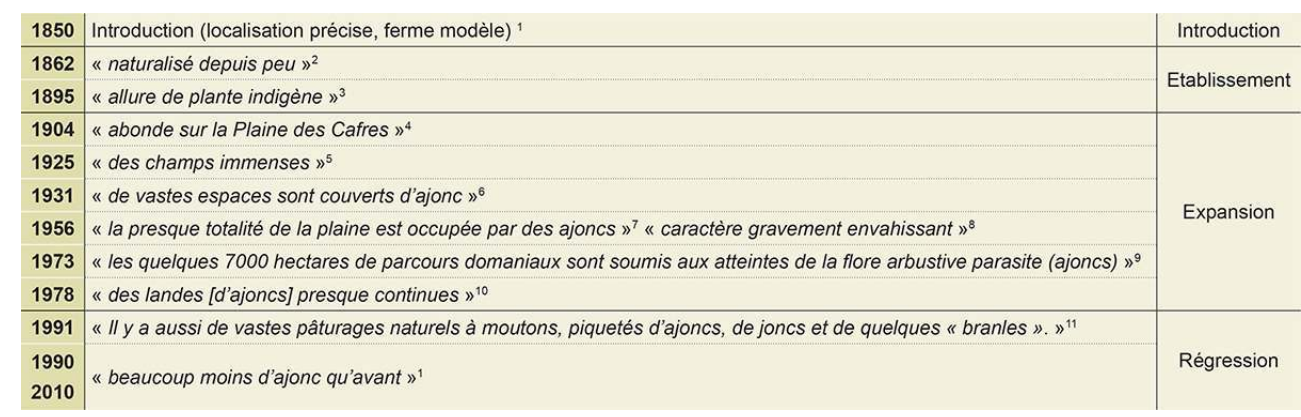

Sources : 1- Entretiens semi-directifs auprès des éleveurs et des responsables agricoles ; 2 - Jacob de Cordemoy [1862] 2004 ; 3- Jacob de Cordemoy 1895 ; 4- Musée colonial de Marseille (France) 1904; 5- Société nationale d'acclimatation de France 1925; 6- May \& Verdat 1931 ; 7- Institut de recherche scientifique de Madagascar 1956 ; 8- Benda 1956 ; 9- Université de la Réunion 1973 ; 10- Lavergne 1978 ; 11- Raunet 1991

32 Le processus d'invasion de l'ajonc sur la Plaine des Cafres comprend donc une phase d'introduction entre 1850-1883, une phase de naturalisation entre 1850-1895, une phase d'expansion entre 1900-1980, concomitante, dans les dernières décennies, à l'apparition de nouveaux foyers, suivie d'une phase de régression des densités sur la Plaine des Cafres entre 1980-2010, mais d'une augmentation des surfaces colonisées dans les foyers plus récents (Figures $2 \mathrm{a}, 2 \mathrm{~b}$ et $2 \mathrm{c}$ ). Le pic de présence de l'ajonc sur la Plaine semble avoir été atteint dans les années 1970-1980, ce qui est confirmé par les entretiens auprès des habitants, éleveurs et responsables agricoles.

\section{Facteurs explicatifs de la phase d'expansion (1900-1980)}

33 L'expansion de l'ajonc dans la Plaine des Cafres est possible parce que les conditions climatiques et pédologiques lui sont favorables et du fait de la perte de ses ennemis 
naturels, notamment les prédateurs de graines. Elle a aussi été favorisée parce qu'il n'y a pas eu de transfert de savoir-faire entre la France métropolitaine et La Réunion pour le contrôle de l'expansion de cette espèce pionnière (Atlan et al. 2015). Nous allons voir dans cette partie que l'expansion est aussi due aux modifications d'usages du sol et à l'apparition croissante de friches ou "vieilles jachères ", favorables au caractère pionnier et rudéral de l'ajonc. Le développement de ces friches, dû à des défrichements et des abandons de terres agricoles, a des causes sociales multiples émanant de mesures sociopolitiques, prises à l'échelle régionale et nationale et remontant, pour certaines, à plusieurs siècles. L'interaction entre les éléments naturels et sociaux favorisant chacune des étapes de la dynamique de l'ajonc à la Plaine des Cafres est présentée Figure 4.

Figure 4 : Facteurs explicatifs des phases d'expansion, de dispersion et de régression de l'ajonc d'Europe à La Plaine des Cafres entre 1900 et 2010

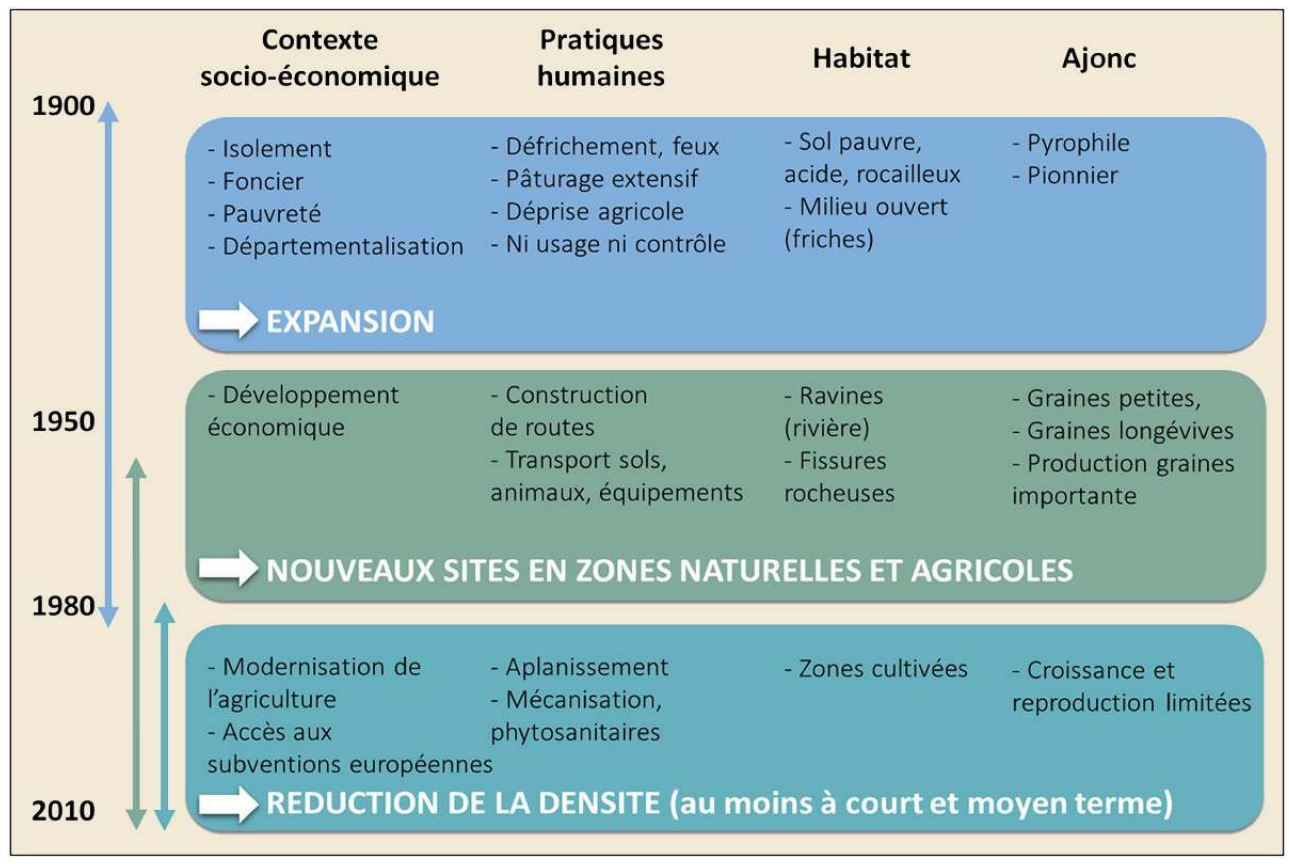

\section{Défrichement et incendies}

Le climat rude (hiver froid et humide), la pauvreté du sol et les difficultés techniques et économiques de l'élevage dans la Plaine des Cafres ont conduit à l'abandon des terres agricoles, très peu de temps après l'attribution des concessions (Bois Joli Potier 1871, Defos de Rau 1960, Bertile 1978, Fontaine 1988). La population restante, les «petits blancs des Hauts», est majoritairement désargentée et paupérisée (Dalama 2006). Oubliés des politiques de développement de l'île jusqu'aux années 1980, ils n'ont souvent eu d'autres solutions, devant la pauvreté des sols et le manque de moyens techniques et économiques, que de multiplier les défrichements, par coupes ou brulis, pour pouvoir cultiver leurs aliments de subsistance. Les incendies ont été très fréquents au moins depuis le XIX ${ }^{e}$ siècle et jusqu'à récemment (Textor De Ravisi 1850, Defos de Rau 1960, Lechartier 2009). Ils permettaient de créer des zones de pâturage pour l'élevage extensif (ONF 1987, Delcour 1993). Les incendies ont contribués à ouvrir le milieu mais aussi à lever la dormance des graines des ajoncs, qui sont parmi les premières à germer. L'ajonc a 
ainsi pleinement profité des pratiques de culture locale pour s'étendre sur la plaine (Blanfort 1996).

\section{Les transitions agricoles}

À partir de la fin du XIXe la transition agricole rapide et massive de l'élevage extensif à la culture et distillation du géranium a occasionné des friches par au moins deux voies. Premièrement, les terrains ouverts utilisés pour l'élevage, bordés d'ajoncs, se sont retrouvés à l'abandon (Defos de Rau 1960). Deuxièmement, la culture du géranium a entraîné des défrichements considérables dans les hauts pour la plantation et pour la collecte de combustible à alambics (Kopp 1937). En 1970-1975, l'arrêt de la culture du géranium a accentué encore l'expansion de l'ajonc car les terrains défrichés pour le géranium ou pour le bois de chauffage ont alors été laissés à l'abandon (Defos de Rau 1960, Benoit 1991).

\section{Le parcellaire traditionnel et la gestion du foncier}

Depuis le début de la colonisation de La Réunion (XVII ${ }^{\mathrm{e}}$ siècle), le foncier agricole de lîle a été découpé «du battant des lames au sommet des montagnes" et ces lanières ont été attribuées à de grands propriétaires (Lavaux 1998). Au fil des générations, ces lanières ont été divisées par héritage dans le sens de la longueur, ce qui a conduit à des terrains très étroits (parfois quelques mètres). Malgré un redécoupage des terrains agricoles au moment de l'attribution des concessions au xIx ${ }^{\mathrm{e}}$ siècle (Textor De Ravisi 1850, 1852), certaines de ces lanières subsistent et, n'étant adaptées ni à une mise en culture ni au pâturage, sont souvent abandonnées et se sont enfrichées (Dalama 2006). En 1990, d'autres facteurs liés à la gestion du foncier maintiennent, voire favorisent, l'abandon des terres : l'indivision des terrains depuis deux à trois générations, la non identification des propriétaires, les difficultés de suivi de foncier et les erreurs de cadastre (Guellec 1992).

\section{La grande déprise agricole}

37 La départementalisation de La Réunion en 1946 apporte des moyens humains et financiers considérables, mais les premières étapes du développement apparaissent à partir des années 1960 exclusivement sur la frange littorale de l'île, les Bas, et aucunement dans les Hauts (dont la Plaine des Cafres) (Benoit 1991, Dalama 2006). Ces masses financières et le besoin de main d'œuvre dans les Bas vont bouleverser les structures sociales et économiques, notamment en créant un exode rural important des Hauts vers les Bas et donc une forte déprise agricole dans la Plaine des Cafres (Defos de Rau 1960).

\section{Facteurs explicatifs de la phase de régression (1980-2010)}

La régression de l'ajonc sur la période 1980-2010 est majoritairement liée à la reconquête des Hauts pour un élevage plus productif basé sur le modèle européen (figure 4). Cette modernisation de l'élevage a été une des mesures phare du Plan d'Aménagement des Hauts (PAH) de La Réunion, qui a pris son essor à partir des années 80 suite à une forte implication des élus, des administrations, des collectivités locales, de l'ONF, des socioprofessionnels sur le terrain (Guellec 1992, Dalama 2006). Le développement de l'élevage est alors reconnu comme un enjeu clé dans l'aménagement du territoire et dans 
la structure socio-économique de l'île (Benoit 1991). Les friches précédemment délaissées et abandonnées sont revalorisées et sous contrôle. L'ajonc est arraché, coupé, traité. Les jeunes plants n'atteignent que rarement l'âge adulte et n'ont souvent pas le temps de se reproduire.

\section{Une efficacité technique croissante et un accès aux aides agricoles européennes}

Le développement de l'élevage sur les plans techniques et économiques bénéficie du soutien de politiques volontaristes et de financements importants de l'Europe, l'Etat, la Région, etc. À partir des années 1980, des études technico-économiques vont être conduites par différents acteurs du monde agricole (ARP, CA974, Centre de coopération Internationale en Recherche Agronomique pour le Développement CIRAD), soutenus par les collectivités locales et des banques afin de mettre en place des itinéraires techniques et permettre la mise en place de prairies et la mécanisation sur de grandes surfaces . Le directeur de l'ARP illustre par ces propos le lien entre cette efficacité technique et la diminution de l'ajonc dès les années 1980

" les bulldozers sont arrivés, et en quelques années, en deux décennies, on a tout foutu par terre. À cette époque, sur l'ensemble des Hauts, on créait 600-700 ha de prairies chaque année, c'est pas mal. De l'ajonc, il y en a beaucoup moins qu'avant, on peut encore en trouver dans quelques friches, mais il y en a bien moins qu'avant, il y a eu beaucoup de défrichements, de mise en place de pâturages, donc l'ajonc c'est plus vraiment un problème » (2013).

Dès les années 1990, la majorité des zones à vocation pastorale est mise en valeur et exploitée. Aujourd'hui, pour les éleveurs enquêtés installés depuis plusieurs dizaines d'années, la régression des ajoncs résulte clairement de leur action technique et « acharnée " pour réduire la surface occupée par cette espèce : "C'est un travail de 30 ans quand même. [...] on les surveille de près" (un éleveur, 2013). Les associations (ARP) et institutions agricoles (CA974, Direction de l'Alimentation, de l'Agriculture et de la Forêt DAAF) ont un discours concordant avec celui de ces éleveurs : l'ajonc n'est pas, ou plus, un problème sur la plaine depuis au moins 10-15 ans du fait des itinéraires techniques mis en place (ex: Blanfort 1996, Barbet-Massin et al. 2003), des moyens techniques disponibles et du travail des éleveurs. Pour le directeur de l'ARP, l'ajonc « fait partie des ligneux qu'ils [les éleveurs] ont l'habitude de gérer ", le responsable élevage de la chambre d'agriculture conclue qu' "ils le maitrisent [...] seul et individuellement", enfin pour un responsable phytosanitaire de la DAAF, «l'ajonc est sous contrôle» (2013). Par ailleurs, au cours des années 1980, le passage du statut de « propriétaire d'animaux » à celui « d'éleveur individuel » donne à ces derniers accès aux aides financières et techniques nationales et européennes, en particulier pour les défrichements mécaniques (Raunet 1991).

\section{Une réforme foncière favorable}

41 Le PAH a reposé sur la mise en valeur pastorale de "terres incultes ou/et dégradées ", souvent colonisées par l'ajonc. Après une législation en 1981, la politique des terres incultes se développe dans les années 85-88 et se renforce suite au décret du 26 juin 1990 : les terres qui ne sont pas cultivées seront rachetées par la Société d'aménagement foncier et d'établissement rural (Safer) et redistribuées à des personnes souhaitant se lancer dans la culture ou l'élevage ou agrandir leur exploitation (Guellec 1992). Par ailleurs, sur les terrains qu'elle attribuait aux éleveurs, la Safer apportait un soutien logistique et financier : elle pouvait financer la location d'engins agricoles pour aplanir 
les terrains et l'achat de désherbant pour détruire les repousses et plantules d'ajoncs. Un éleveur témoigne du lien entre cette politique et la diminution des ajoncs :

«Les terrains étaient abandonnés, les propriétaires faisaient rien, mais tu pouvais rien faire dessus non plus, c'était pas à nous. Après la Safer a pris ces terrains-là, a revendu aux agriculteurs. Le terrain où est mon frère là, c'était un grand terrain qui était abandonné depuis je ne sais combien d'années, était en zépine, et la Safer a partagé, en 4-5 éleveurs [...]. Avec les pâturages que nous avons faits, au Piton Bleu n'avait que ça, mais ça a été détruit » (2013).

\section{Dispersions et apparition de nouvelles populations}

Il existe actuellement une dizaine de foyers d'ajonc autour de la Plaine des Cafres, notamment dans le Massif du Volcan (Figure 2c), tous postérieurs aux années 1960-1970, phase d'expansion maximale des ajoncs sur la plaine. Les témoignages et l'analyse des activités anthropiques pendant cette période permettent de formuler des hypothèses sur les modes de dissémination des graines.

\section{Dispersion liée à l'agriculture}

Jusqu'aux années 50 environ, les troupeaux de bovins et d'ovins (plusieurs milliers de bêtes) erraient librement dans la Plaine des Cafres et dans le Massif du Volcan, puis, dans le courant des années 60 , un système de transhumance a été mis en place par l'ONF : les troupeaux étaient déplacés tous les 2 à 3 mois sur 5 zones dans le Massif du Volcan, en plus de la Plaine des Cafres (Defos de Rau 1960, ONF 1987, Delcour 1993). Les ovins en particulier sont reconnus comme un facteur de dispersion sur plusieurs dizaines de kilomètres de nombreuses graines dans leur toison de laine (Dutoit et al. 2003) dont l'ajonc (mis en évidence en Nouvelle-Zélande par Moss 1959). De fait, sur 4 des 5 zones de transhumance, l'ajonc est présent. Toutefois, d'après les entretiens auprès des éleveurs et des gestionnaires, l'ajonc serait majoritairement arrivé dans ces nouveaux foyers à partir de 1980 , période où les pratiques agricoles se sont modifiées dans le cadre de la modernisation de l'agriculture avec l'augmentation des transports de fourrage, d'animaux et les échanges de matériel dans les coopératives et au sein des familles.

\section{Dispersion liée au transport de matériaux issus des carrières}

Les fourrés parmi les plus denses de la Plaine des Cafres accueillent la principale carrière de scories basaltiques (pouzzolane) de La Réunion. Les scories, fragments de lave avec une structure alvéolaire pouvant retenir des graines, sont utilisées dans le domaine de l'aménagement (notamment des routes), de la construction et de la décoration extérieure. Elles sont reconnues comme «le produit péi [produit du terroir] par excellence, local et naturel » et sont par conséquent très utilisées tant par les collectivités locales et les gestionnaires que par les entrepreneurs privés et les particuliers. Au cours du PAH (à partir des années 1980), les engins d'excavation pouvaient être mobilisés sur plusieurs carrières, ce qui peut expliquer la présence de l'ajonc sur la quasi-totalité des anciens sites de carrières de scories. Dans plusieurs cas, l'utilisation de scories a précédé de peu l'arrivée des ajoncs. 


\section{Dispersion par les ravines} transport par l'eau dans les réseaux de ravines (Beltran \& Pausé 2012). Ce phénomène entraîne des fronts de dispersion localisés le long des cours d'eau très facilement identifiables. Dans le Massif du Volcan (comme dans celui du Maïdo, cf. ci-dessous) les premiers ajoncs ont été introduits de manière anthropique, mais l'expansion géographique s'est accélérée de manière spectaculaire lorsque les premiers ajoncs ont rejoint le réseau de ravines (figure 2c). Le transport des graines par l'eau a aussi pu augmenter dans le cadre de la valorisation de la laine de moutons au $\mathrm{xx}^{\mathrm{e}}$ siècle: les femmes se réunissaient au bord des ravines pour nettoyer dans l'eau la laine fraichement tondue juste avant l'été austral, au moment du pic de fructification de l'ajonc (Lechartier 2009).

\section{Dynamique spatio-temporelle mystérieuse de l'ajonc dans le Massif du Maïdo}

\section{Phase d'introductions de l'ajonc sur le Massif du Maïdo}

\section{Les premières descriptions de l'ajonc sur le Massif}

Le Massif du Maïdo accueille actuellement la troisième plus grande population d'ajoncs dans le milieu naturel protégé. La première trace écrite de la présence des ajoncs date de 1948. Il est mentionné dans le récit d'une expédition des techniciens de l'IGN (Lougnon 1977a) :

«[...] les techniciens de l'IGN sont venus à la Réunion en 1948. [...] Quand le topographe de l'IGN arriva au Maïdo, où il planta sa mire, il avait dû traverser des champs d'ajoncs aux épines terribles qui vous infligent une puissante gratelle. Les porteurs qui l'accompagnaient, forts sensibles à ces épines, déclarèrent que c'était le Piton des « épinards » (en raison des épines) [...]. »

Sur la carte IGN de La Réunion publiée en 1957 (IGN 1957), le toponyme de l'actuel Piton Maïdo est « Piton des Epinards », un des noms local de l'ajonc. Avant 1948-1955, très peu de gens ont fréquenté le Massif du Maïdo (Souffrin 1995) ; les rares descriptions écrites ne permettent pas de borner précisément l'introduction et la naturalisation de l'ajonc. Des informations complémentaires ont été collectées par entretiens en 2013 auprès des habitants d'un village du cirque de Mafate (Roche Plate) connecté au Massif du Maïdo par un petit sentier dans les remparts (Figure 2d). Depuis le bord du Massif du Maïdo (2200 m d'altitude) ce village ( $800 \mathrm{~m}$ d'altitude) est visible, et réciproquement. Plusieurs habitants font état de la présence d'ajoncs le long du sentier qu'ils empruntaient pour rejoindre le Massif du Maïdo, au moins à partir de 1930. Un habitant d'une cinquantaine d'années se souvient que pendant son enfance « on en voyait déjà beaucoup le long des sentiers. [...] mes parents se piquaient avec le sapan en empruntant les sentiers [vers 1945] », tandis qu'un ancien (94 ans) raconte «Quand on était petit [vers 1930], il y avait déjà des sapans sur le sentier ».

D'autres indiquent que l'ajonc était «partout sur la planèze [Massif du Maïdo] » au moins depuis les années 1940, mais cela est en contradiction avec les écrits de plusieurs botanistes (Cadet 1974, 1977, Lavergne 1978, Channg Yok 1992), et donc peu probable. Il est, en revanche, possible que, dès 1940, tout le bord du rempart visible depuis le village ait été occupé par l'ajonc; nous allons y revenir. 


\title{
Motivations de l'introduction
}

\begin{abstract}
dans le Massif du Maïdo. En revanche, plusieurs enquêtés (acteurs de l'environnement et habitants) ont émis des hypothèses. Nous sommes donc parties de ces hypothèses et nous avons essayé de les réfuter ou de mettre en évidence des éléments convergents les renforçant.
\end{abstract}

\section{La création de la piste et de la route}

50 L'hypothèse la plus partagée par les gestionnaires travaillant dans la zone était d'attribuer l'arrivée de l'ajonc sur le Massif à la création de la route et plus particulièrement du parking dans les années 1980 (Figure 2e). Des graines d'ajonc auraient été introduites en même temps que les matériaux (scories) et les engins utilisés pour sa construction. Cependant, la première piste a été créée en 1954-55 et a ensuite été bitumée dans les années 1969-71 alors qu'il y avait déjà de l'ajonc dans les années 1930. De plus, ces aménagements ont essentiellement été réalisés avec des matériaux en provenance d'une commune des Bas de l'île où l'ajonc est absent (Lougnon 1977b). Cette hypothèse est donc réfutée.

\section{Le pâturage extensif}

51 Certains gestionnaires émettent l'hypothèse d'une introduction de l'ajonc liée à des actes volontaires (nourriture sur pied) ou involontaires (graines dans le fourrage) par les éleveurs effectuant du pâturage extensif. Effectivement, dès la fin du XIX ${ }^{e}$ siècle et jusqu'à aujourd'hui, des troupeaux de bovins pâturaient de manière extensive et parfois illégale dans le Massif du Maïdo (E.D. 1896, Lustenberger 2009). À la fin du XIx ${ }^{\mathrm{e}}$ siècle, il a même été proposé de mettre en place un élevage bovin dans ce massif. Le sol volcanique est décrit comme «le meilleur sol à lait ", où «il serait facile d'y semer les semences des plantes qui croissent sur le plateau des Salers " (E.D. 1896). Toutefois, l'ajonc ayant peu été utilisé comme fourrage pour les bovins dans la Plaine des Cafres (Atlan et al. 2015), il est peu probable qu'il ait été planté pour cet usage dans le Massif du Maïdo. Une introduction involontaire lors de transports de fourrage semble également peu probable car à cette époque les transports entre la Plaine des Cafres et le Massif du Maïdo étaient rares.

\section{La création d'obstacle au déplacement}

Un habitant du village de Roche Plate suggère que l'ajonc a été planté volontairement au début du sentier " pour déranger les esclaves réfugiés dans le cirque [de Mafate], pour bloquer le chemin ». De fait, que ce soit contre ces esclaves en fuite ou contre des "petits blancs des Hauts » qui ont par la suite également été vivre dans le cirque (Dalama 2006), plusieurs groupes ont été opposés à l'occupation de ce site par des habitants. Outre les chasseurs d'esclaves au $18^{\text {ème }}$, le Service Forestier (ancêtre de l'ONF) a voulu chasser vers 1874 les habitants du cirque afin de procéder à son reboisement (Bonniol \& Benoist 1994). Si l'histoire d'une introduction par des éleveurs de bovins est croisée avec celle d'une clôture défensive contre les habitants du cirque, il est possible de formuler une nouvelle hypothèse, appuyée sur des commentaires de 1896: "Les habitants de Mafate [...] escaladaient le rempart de la rivière des galets, et venaient abattre une bête dans le troupeau qui 
s'était égarée vers la glacière [une zone du Massif du Maïdo].» (E.D. 1896). On peut ainsi supposer que des éleveurs de bovins ont planté de l'ajonc en haut du sentier et du rempart de Mafate pour empêcher les habitants du cirque de venir tuer et récupérer leurs animaux. En somme, au cours de l'histoire, plusieurs personnes ont pu avoir intérêt à disposer une plante épineuse à l'entrée du sentier et en haut du rempart pour réaliser une clôture défensive, bien que cela ne soit pas étayé par les archives. L'hypothèse d'une clôture défensive est d'autant plus vraisemblable que c'était l'un des usages en cours à cette époque dans la Plaine des Cafres (et globalement dans les colonies européennes), et qu'aujourd'hui encore une bande d'ajoncs particulièrement dense est nettement visible le long du rempart de Mafate dans les cartographies de la plante (ex : Payet 2012).

\section{Dynamique spatio-temporelle sur le Massif du Maïdo}

\section{Une expansion en deux temps}

Les informations émanant des documents et des entretiens sont peu nombreuses, mais les localités mentionnées sont souvent précises et permettent de retracer l'expansion de l'ajonc dans le Massif du Maïdo de 1930 à 2014.

De 1930 à 1980-1990, l'ajonc semble être resté cantonné à la partie supérieure du Massif du Maïdo (Figure 2e). Sa propagation, de proche en proche, a probablement été majoritairement due à la dispersion naturelle des graines par éjection lors de l'ouverture des gousses dans un rayon de quelques mètres autour de la plante mère (Norambuena et al. 2000). Ce cantonnement est attesté par le recoupement de plusieurs données: (i) les botanistes Rivals $(1952)$, Cadet $(1974,1977)$ ne mentionnent respectivement pas et très peu l'ajonc dans leurs études dans ce massif; l'ethnobotaniste Lavergne en 1980 ne mentionne l'ajonc que sur le bord du rempart; (ii) l'écrivain-journaliste Jacques Lougnon, spécialiste de la zone, ne mentionne également l'ajonc qu'au niveau du rempart et du parking jusqu'à la fin des années 1970 (Lougnon 1977b); (iii) un randonneur ayant parcouru le Massif dès les années 1980 se souvient surtout des ajoncs «très nombreux sur tout le bord du rempart » (2013).

À partir des années 1990, l'ajonc "envahit certains endroits » dans la partie supérieur du Massif du Maïdo, et « fait son apparition » dès 1900-2010 m d'altitude (Channg Yok 1992), ce qui suggère sa descente vers l'Ouest en direction de la mer. Environ 15-20 ans plus tard, les cartographies de l'ajonc réalisées par l'ONF et le Parc National indiquent une présence quasi-continue de l'ajonc jusqu'à $1750 \mathrm{~m}$ d'altitude sur le Massif du Maïdo (Triolo \& Hoff 2006, Payet 2012). En 2014, l'ajonc est présent sur $14 \mathrm{~km}^{2}$ et forme des fourrés denses quasi-continus sur 2,4 $\mathrm{km}^{2}$ (Figure 2f).

Plusieurs petits foyers ont été observés sur la côte Ouest. Un premier foyer est situé à $1300 \mathrm{~m}$ d'altitude, le long de la route départementale qui rejoint le Maïdo au bord de mer. D'après les entretiens auprès des propriétaires des terrains concernés, l'ajonc est présent depuis 2010-2011 et a germé dans les remblais de terre en provenance du Maïdo. Deux autres foyers d'ajonc se développent également dans des remblais de terre, sur une route nationale et sur une piste forestière, à des altitudes inférieures à celle de la population principale (Figure 2f). Contrairement à la Plaine des Cafres, il n'y a pas eu d'apparition de gros foyers en périphérie du foyer principal. 
Figure 5 : L'ajonc d'Europe en fleur au niveau du point du vue du Maïdo sur le cirque de Mafate

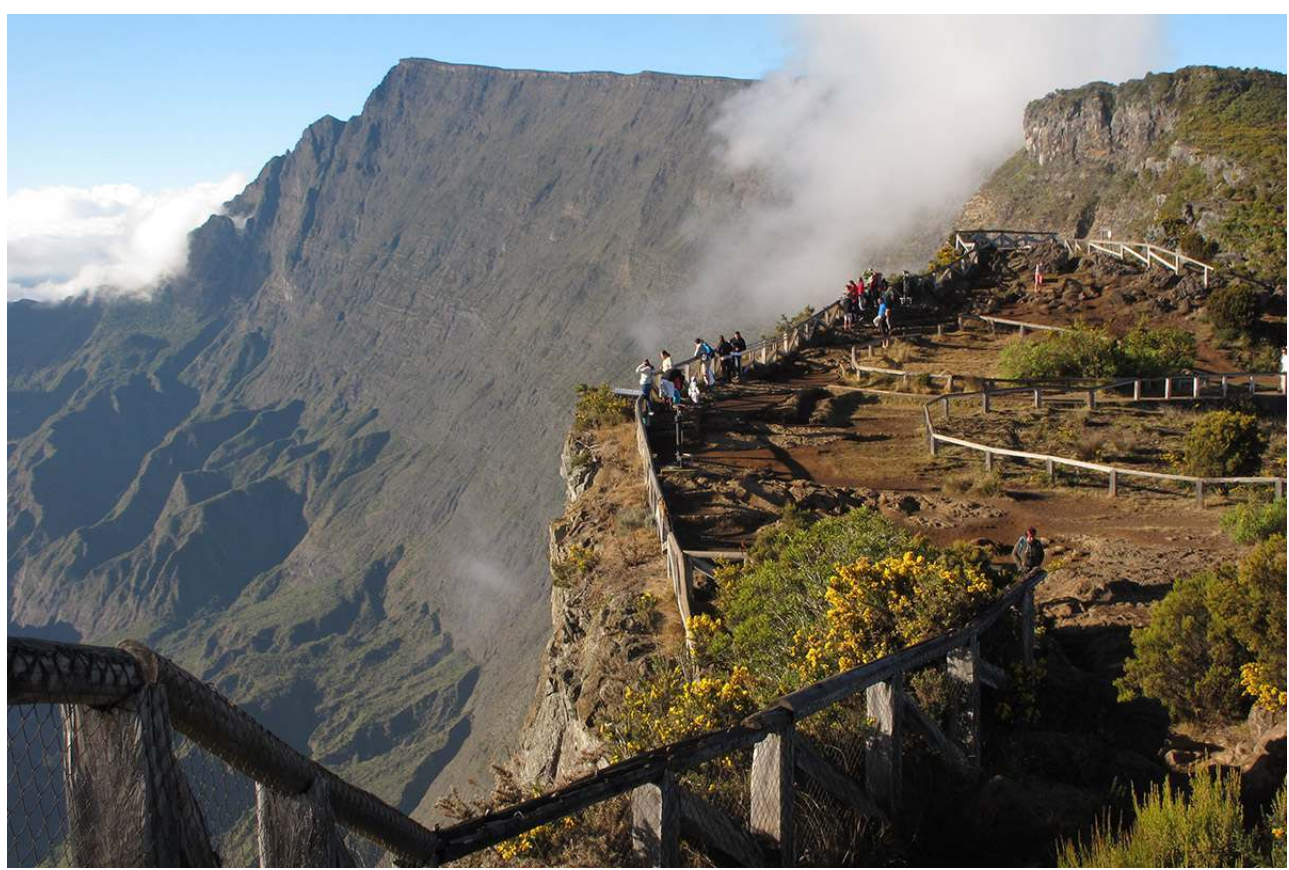

Cliché : N. Udo 2013

L'expansion de l'ajonc sur le Massif du Maïdo s'est donc faite en deux temps : un premier temps, plutôt lent, pendant lequel l'ajonc s'est étendu de proche en proche (1930'-1980'), et un second temps, plutôt rapide (1980-2012), pendant lequel l'ajonc s'est installé en fourrés denses jusqu'à $1750 \mathrm{~m}$ d'altitude et s'est dispersé dans de nouveaux petits foyers sur les plus basses altitudes de la côte Ouest. L'ajonc ne semble pas avoir poursuivi sa descente vers le cirque de Mafate : en 1930 il atteignait déjà probablement la limite de sa niche climatique favorable.

\section{Facteurs explicatifs de l'expansion des années 1980 à aujourd'hui}

\section{Les bovins divagants}

Le Massif du Maïdo est fréquenté au moins depuis la fin du XIX ${ }^{e}$ siècle et jusqu'à aujourd'hui par des bovins élevés de façon très extensive dans le milieu naturel. Cette pratique s'est particulièrement développée à partir de la seconde moitié du $\mathrm{xx}^{\mathrm{e}}$ siècle, avec une forte accentuation depuis les années 1970-1980, même si les chiffres actuels varient de 300 à 2000 selon les acteurs en 2009 (Lustenberger 2009). Ces bovins broutent les jeunes pousses des espèces indigènes plantées par l'ONF et sont suspectées de faire la même chose dans le milieu naturel. Certains gestionnaires de l'environnement défendent l'idée que ces bovins faciliteraient l'expansion de l'ajonc en dispersant des graines dans la boue des pattes ou via les déjections et/ou en retournant la terre conduisant à la germination des graines et/ou en facilitant la compétition de l'ajonc grâce à une consommation importante des plantules d'espèces indigènes. Pour autant, aucun protocole n'a encore été mis en place pour tester ces hypothèses et les jeunes pousses d'ajonc semblent aussi broutées par les bovins que les pousses d'indigènes (Lustenberger 2009). 


\section{Les incendies}

Le Massif du Maïdo a fréquemment subi des incendies de grande ampleur. D'ailleurs, le nom Maïdo signifie «terre brûlée » en malgache (Lougnon 1977a, Lavergne 1980). Les botanistes Rivals $(1952)$, puis Cadet $(1974,1977)$ abordent à plusieurs reprises ces incendies périodiques, essentiellement d'origine anthropique. Les motivations à l'usage du feu dans le massif sont multiples, mais deux principales ont été relevées par Lustenberger (2009): la création de zones de pâturage et des mesures de représailles, auxquelles nous ajoutons la pyromanie, comme pour l'incendie de 2010. Entre 1970 et 2014, cinq incendies ont eu lieu sur la totalité ou une partie de l'actuelle zone d'ajoncs. Le haut du sentier et le bord du rempart où l'ajonc est particulièrement dense et présent depuis les années 1930 ont subi trois incendies sur cette période. La très grande majorité de la surface occupée aujourd'hui par les ajoncs a été brûlée au moins une fois de 1970 à 2014. L'incendie de $2010\left(7,6 \mathrm{~km}^{2}\right)$ est le dernier incendie le plus remarquable ; la quasitotalité de la population d'ajonc a alors été brûlée. À la suite de cet incendie, du fait de sa capacité à germer et à donner des rejets de souche (Figure 6), l'ajonc a eu tendance à devenir plus dense là où il était déjà présent, et de nouveaux espaces ont été occupés.

Figure 6 : L'ajonc d'Europe deux mois après l'incendie d'octobre 2010 au Maïdo

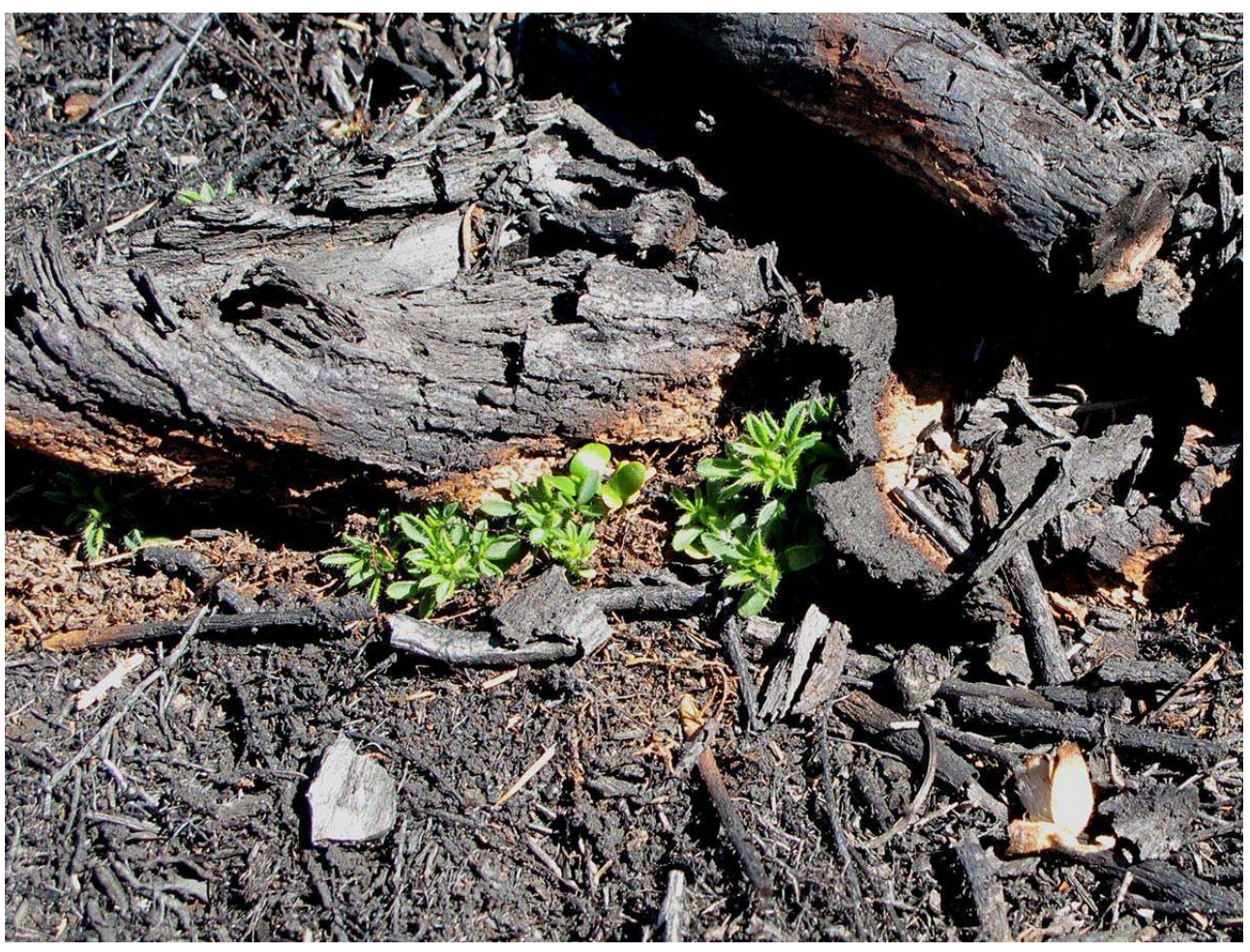

Germinations

Cliché : Atlan 2010 


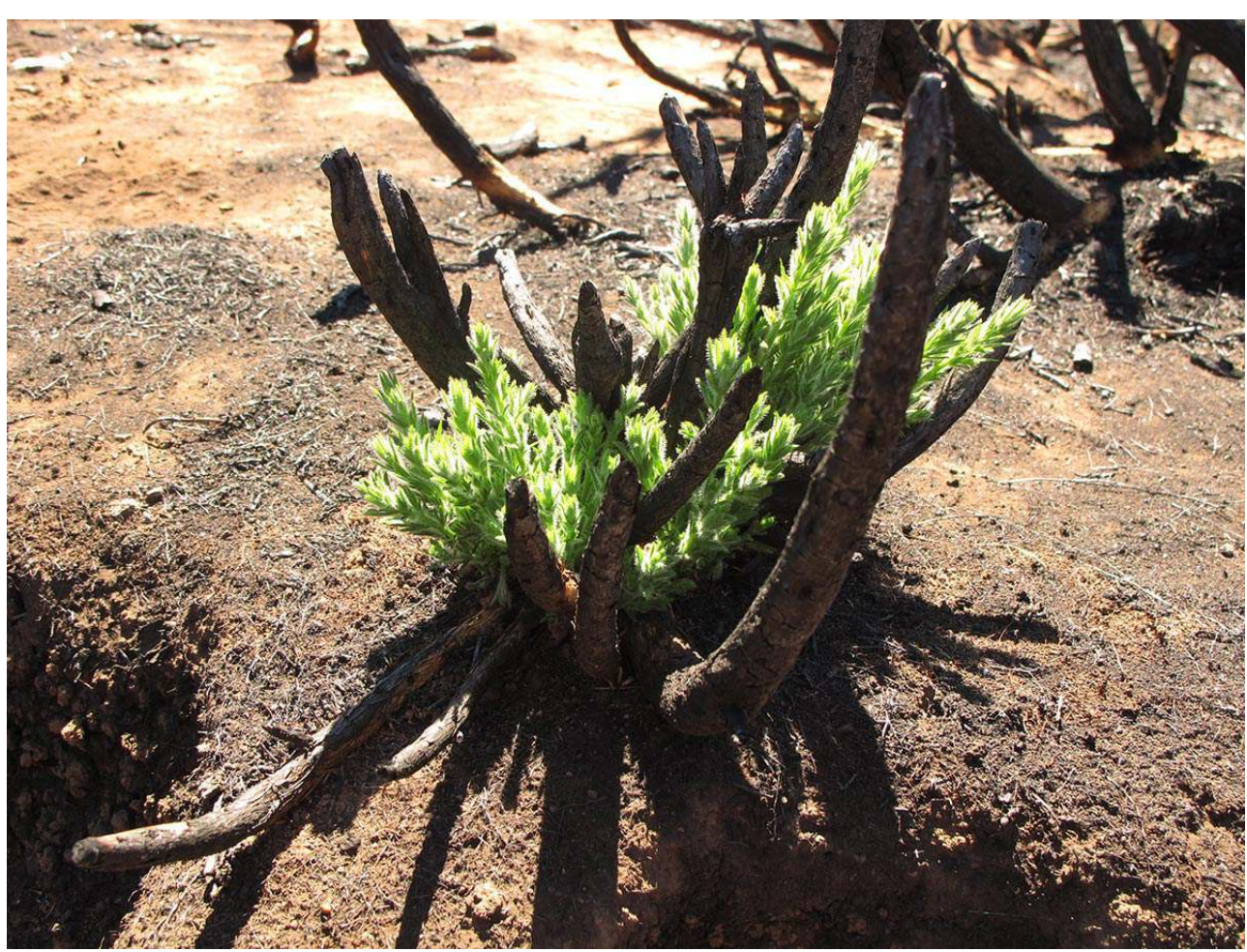

Rejet de souches

Cliché : Atlan 2010

\section{Les pare-feu et les pistes}

\begin{abstract}
par l'ONF (années 50). Ces pare-feu s'étendent sur plusieurs kilomètres et font environ 10 mètres de large. Ils créent de larges espaces ouverts propices au développement des espèces pionnières et particulièrement des ajoncs. L'entretien des pare-feu implique l'arrachage régulier de toute végétation. La difficulté d'accès de ces pare-feu, les restrictions budgétaires régulières de l'ONF (depuis 2000-2005) et les controverses autour de leur utilité (2010-2014) ont conduit à une gestion moins efficace, voire une nongestion, des pare-feu du massif favorisant l'ajonc qui peut ainsi recouvrir d'importantes surfaces. Les pistes forestières ouvertes dans la végétation par l'ONF ou par les pompiers posent des problèmes similaires.
\end{abstract}

\section{Les ravines et les fissures}

61 Le Massif du Maïdo est recouvert d'une dalle rocheuse basaltique, traversée par plusieurs ravines plus ou moins connectées formant un réseau complexe d'écoulement des eaux. La zone d'introduction de l'ajonc supposée (entrée du sentier et bord de rempart) n'est pas directement reliée à des ravines. En revanche, dès que l'ajonc a atteint le rempart, environ un kilomètre plus au sud, il est entré en connexion avec plusieurs grandes ravines, favorisant son extension vers les bas de l'île, à partir des années 1970-1980. Il a alors formé plusieurs foyers denses le long de ravines, parfois de plus de $2 \mathrm{~km}$ de long et sur plus de $500 \mathrm{~m}$ de dénivelé. De plus, à une échelle beaucoup plus petite, la dalle rocheuse du Massif du Maïdo est parcourue de nombreuses fissures (quelques centimètres ou dizaines de centimètres de large) qui représentent également des micro-couloirs de 
dissémination des graines d'ajonc. En période de fructification, plus de 500 graines ont été comptabilisées dans une cavité le long d'une fissure, sur seulement $200 \mathrm{~cm}^{2}$ (Figure 7).

Figure 7 : Graines d'ajonc d'Europe dans une cavité le long d'une fissure de la dalle basaltique du Massif du Maïdo

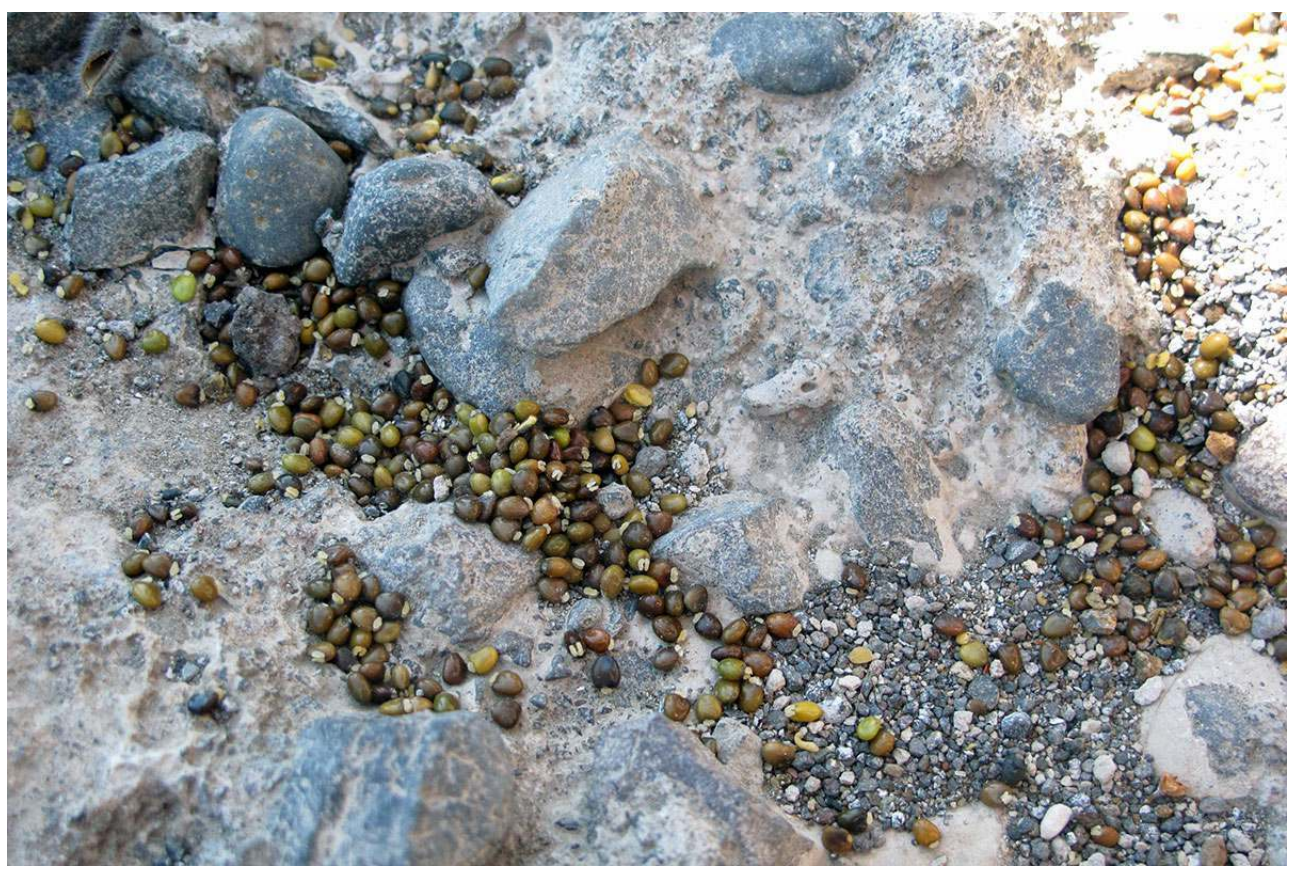

Cliché : N. Udo 2013

\section{L'absence d'une phase de régression}

Depuis 2003, des actions de luttes mécanique et chimique contre l'ajonc sont effectuées par l'ONF dans ce massif. Le lancement de ces actions, planifiées dans une stratégie de lutte (Triolo \& Hoff 2006) est concomitant à la volonté d'intégrer ce massif dans le cœur du Parc National de La Réunion (créé en 2007). En 2010, le vaste incendie qui a brûlé la population d'ajonc et par là même a favorisé sa germination et sa régénérescence, a conduit à un investissement important des agents du Parc National aux côtés des agents de l'ONF : de nouvelles stratégies de luttes sont négociées et des actions de "détection précoce et éradication » sont programmées dans l'objectif de ralentir l'expansion sur les fronts et d'éviter de nouveaux foyers. Toutefois, les techniques utilisées dans les espaces agricoles ne peuvent être utilisées dans un milieu naturel protégé en raison du nombre important d'espèces indigènes se développant en maillage avec l'ajonc et le champ des techniques de lutte envisageables est restreint. Couplé à la réduction constante des moyens humains et financiers alloués à cette lutte, les actions menées n'ont pu empêcher ni la densification de l'ajonc dans les zones où il était déjà présent ni son expansion dans les zones difficiles d'accès, telles que le rempart et les ravines. Ainsi, contrairement à ce qu'on constate dans la Plaine des Cafres, il n'y a, à ce jour, pas de phase de régression dans le Massif du Maïdo. 


\section{Synergie des facteurs naturels et humains tout au long du processus invasif}

\section{La reconstitution des dynamiques invasives à l'aide d'une méthode transdisciplinaire}

63 L'histoire de la dynamique spatio-temporelle d'invasion de l'ajonc à La Réunion a pu être retracée sur près de 200 ans grâce à la complémentarité et aux recoupements des informations collectées dans les documents d'archives, la littérature grise et académique, les cartographies, et les entretiens semi-directifs auprès des acteurs de l'environnement, des agriculteurs et des habitants. La dynamique du processus d'invasion retracée à l'aide de ces données est cohérente avec la dynamique écologique habituellement décrite des espèces exotiques envahissantes (Mack et al. 2000, Blackburn et al. 2011). Le degré de précision atteint est très important (à l'échelle temporelle de la dizaine d'années et à l'échelle spatiale de l'ordre du kilomètre), y compris dans les zones inhabitées et peu fréquentées, comme le Massif du Maïdo.

Puisqu'elle s'est avérée adaptée à la reconstitution de la dynamique invasive de l'ajonc à La Réunion, nous proposons d'inscrire cette méthode transdisciplinaire aux côtés de celles plus couramment utilisées en biologie pour répondre à ce genre de questions, à savoir les marqueurs moléculaires (Williams et al. 2005, Guggisberg et al. 2012), l'étude des parts d'herbier (ex: Delisle et al. 2003, Chauvel et al. 2006, Harris et al. 2007, Lavoie et al. 2007, Aikio et al. 2010), les comparaisons cartographiques, d'échantillonnages ou d'inventaires (ex: Brunzel et al. 2009, Butcher et al. 2014). L'intérêt de multiplier les méthodes pour cerner l'histoire des processus invasifs est d'autant plus fort que chacune a ses limites : les marqueurs moléculaires ne sont pas utilisables sur toutes les espèces (et notamment pas sur l'ajonc à cette échelle de temps et d'espace, Hornoy 2012) et ne permettent pas d'atteindre une précision temporelle fine; la compilation des parts d'herbiers souffre d'un biais lié à l'effort de collecte, variable selon les espaces et les époques (Delisle et al. 2003, Chauvel et al. 2006) ; les comparaisons de relevés et d'inventaires ne permettent que rarement de remonter au-delà de 30 à 40 ans. De plus, ces trois méthodes apportent relativement peu d'informations sur les facteurs explicatifs $\mathrm{du}$ processus d'invasion et aucune sur le contexte social, contrairement à celle que nous proposons ici.

Cette méthode n'est pas seulement complémentaire des autres et efficace, elle promeut le partage de connaissances entre acteurs du territoire et est orientée vers l'action. En effet, il s'agit d'une démarche de l'immersion qui invite au dialogue et met en évidence la complémentarité et la diversité des savoirs des particuliers, des gestionnaires et des scientifiques (Larrère \& Larrère 2009 ou 2012, Roué 2009). Elle a ainsi la capacité d'accompagner la co-construction d'une histoire commune sur un territoire commun, de faire en sorte que « le territoire des autres devienne le territoire de tous » (Joutard 1985, cité dans Lizet 2009). En étant produite avec les gens et pensée de manière compréhensive, cette méthode peut participer à la réconciliation, à la confiance partagée et la coopération entre les acteurs. Ensuite, cette co-construction de l'histoire du territoire intégrant des facteurs de nature variée et appartenant à des échelles variées (de l'international à l'organisation familiale) peut favoriser des projets d'action et de cogestion. Sans aller nécessairement jusqu'à une implication de tous les acteurs concernés, 
cette méthode peut aussi permettre d'identifier des mesures pragmatiques de gestion, en agissant sciemment sur plusieurs facteurs facilitateurs du processus invasif.

\section{Les particularités de chaque étape}

Les processus invasifs peuvent se décrire selon quatre étapes majeures: le transport, l'introduction, l'établissement (ou naturalisation) et l'expansion spatiale de l'espèce (Blackburn et al. 2011). À ces étapes majeures, nous ajoutons la phase de régression spatiale. Cette étude a permis d'identifier à chacune de ces étapes les facteurs humains et naturels et leurs interactions, tout comme elle a permis de contextualiser ces facteurs dans une compréhension plus globale intégrant les caractéristiques socio-économiques et culturelles de l'île de La Réunion (Figure 4).

\section{Transport, introduction et établissement}

67 À La Réunion, l'introduction primaire de graines d'ajonc a été volontaire et datée peu avant 1825. L'introduction a été efficiente du fait du maintien de la viabilité des graines pendant le voyage et de l'entretien des plantes dans un jardin cultivé pendant une trentaine d'années. L'ajonc a ensuite été planté volontairement en milieu agricole, autour des années 1850, dans plusieurs fermes de la Plaine des Cafres. Dans cette plaine agricole, les motivations de ces introductions sont en lien direct avec les usages agricoles de l'ajonc dans la zone d'origine et son appréciation symbolique. Toutefois, l'ajonc a été planté par des éleveurs notables présents depuis plusieurs générations sur l'île, et pas - ou pas uniquement - par des colons directement débarqués de métropole, ce qui signifie que la culture de l'ajonc a probablement été prônée sur l'île au milieu du $19^{\text {ème }}$. Dans le Massif du Maïdo, plusieurs éléments, tant cartographiques qu'historiques, convergent vers l'hypothèse d'une introduction volontaire de l'ajonc pour constituer des clôtures défensives, comme dans d'autres régions du monde où il a été introduit (Atlan et al. 2015), mais en réponse à une problématique locale, à savoir l'occupation conflictuelle d'une région montagneuse. Ces éléments attestent que l'introduction de l'ajonc à La Réunion est bien la résultante de facteurs naturels et humains : elle a été motivée par des envies (la proximité d'une plante aimée), des besoins sociétaux et économiques (une nouvelle plante fourragère, une clôture défensive) et des caractéristiques biologiques de la plante (très épineuse, riche en azote, croissance rapide, capacité à pousser sur des sols pauvres).

L'établissement de la plante dans La Plaine des Cafres (entre 1850 et 1900) et dans le Massif du Maïdo (avant 1930) résulte apparemment de facteurs naturels, notamment la capacité de croissance et de reproduction de la plante, sa diversité génétique, les capacités d'adaptation et l'adéquation entre le lieu d'introduction et la niche climatique de l'espèce. Pourtant la motivation sociétale pour le succès de l'établissement et les moyens déployés pour y parvenir ont aussi joué des rôles significatifs : à l'époque des colonies, dès lors que l'introduction d'une plante est volontaire, tout est mis en œuvre pour que la naturalisation réussisse. Ainsi, au XIXe siècle, la distribution de graines d'une même espèce selon un protocole strict le long d'un gradient altitudinal (tous les 200-300 mètres, du littoral jusqu'à la Plaine des Cafres) était organisée par la Société nationale d'acclimatation de France (1875) et a multiplié de fait les chances d'installation des espèces introduites. À la même époque, de façon moins protocolaire mais conduisant aux mêmes résultats, des centaines de milliers de plants, boutures et graines étaient 
distribuées gratuitement aux habitants de l'île depuis le jardin de naturalisation, et des millions cédés à des prix modiques.

\section{Expansion et dispersion} de dispersion. En saisissant l'origine sociale des modifications de l'environnement, l'effort de contextualisation socio-économique, politique et culturel effectué a permis d'aller audelà de la désignation des perturbations anthropiques comme un facteur explicatif de l'expansion de l'ajonc (Rees \& Hill 2001). De fait, cette expansion a été possible grâce à des interrelations de facteurs naturels et humains, jouant des rôles plus ou moins majeurs en fonction des espaces.

zone agricole, de 1900 à 1980, la multiplication et la pérennisation des friches sont entrées en relation avec les caractères pionniers et pyrophile de la plante et sa capacité à s'installer sur des sols pauvres. Ces friches se sont développées sous l'effet de facteurs historiques, socio-économiques et culturels variés: le parcellaire traditionnel qui limite les capacités de culture et d'élevage en découpant les terrains en fins rubans, l'abandon successifs de l'élevage (1900) puis de la culture du géranium (1970), la déprise agricole suite à la départementalisation (1946), l'élevage traditionnel (surtout jusqu'en 1990) et la pratique régulière des incendies (surtout jusqu'en 1980).

Dans les milieux naturels, c'est souvent par des relais, des successions de facteurs de dispersion - naturels et anthropiques - que l'ajonc est arrivé dans les populations actuelles. Ainsi, plusieurs populations d'ajoncs du Massif du Volcan sont le fruit d'une dispersion par des matériaux ou engins agricoles, suivie d'une dispersion par les ravines. De même l'expansion de l'ajonc dans le Massif du Maïdo s'est brusquement accélérée lorsque l'ajonc a atteint les ravines, et que les graines ont pu atteindre des milieux ouverts favorables à une expansion de proche en proche, en particulier les pare-feu, les pistes forestières et les bords de routes. Notre méthode n'a toutefois pas permis de relativiser et de classer par ordre d'importance ces facteurs de dispersion, ce qui pourrait être étudié par des approches quantitatives.

Dans les milieux anthropisés comme dans les espaces plus sauvages, la fréquence des incendies (un incendie au moins par décennie), plus importante que la durée de vie de l'ajonc (20-30 ans) conduit à un rajeunissement régulier des populations qui ne permet pas l'éventuelle succession naturelle qui pourrait conduire au remplacement de l'ajonc par des espèces ligneuses indigènes à vie longue, à l'image de ce qui se passe dans certaines régions de la zone d'origine (Bourlet 1980) ou de Nouvelle-Zélande (Wilson 1994, Williams \& Karl 2002, Wotton et al. 2013).

\section{Régression}

La phase de régression est le plus souvent la conséquence d'une action de lutte résultant des changements de statut des espaces et des espèces et des actions mises en œuvre en réponse à ces statuts. Elle a pour origine un choix de société. La mise en évidence d'une période de régression de la densité des foyers d'ajoncs sur la zone agricole de la Plaine des Cafres, à partir de 1980, amène à conclure qu'il est à présent techniquement possible de contrôler et de gérer l'expansion de l'ajonc dans ce type de milieu (notamment par des travaux de mécanisation, d'arrachage et de traitement chimique de l'ajonc et de mise en 
culture sur de grandes surfaces), quand le contexte culturel, socio-économique, légal et politique l'encourage.

Dans notre cas d'étude, le Plan d'Aménagement des Hauts initié dans les années 1980 et centré sur le développement de l'élevage sédentaire productif a apporté les moyens nécessaires à la régression de l'ajonc sur la plaine: développement des itinéraires techniques et soutien logistique, formation des éleveurs, subventions pour les travaux de mécanisation, prêts avantageux, réforme foncière. La régression est ainsi une question de rapport entre les moyens mis en œuvre et les caractéristiques biologiques de la plante. Ce résultat est en partie en contradiction avec ce qui a pu être mis en évidence auparavant suggérant que l'intensification des milieux agricoles est un des principaux usages du sol responsable du succès des invasions (Vilà \& Ibáñez 2011). En fait, l'effet d'une caractéristique du paysage joue différemment sur le succès des invasions en fonction des espèces. Dans le cas d'une espèce invasive pérenne et ligneuse, une intensification agricole conduit à la régression, mais dans le cas d'une espèce herbacée ou d'une annuelle, une même pratique peut conduire à une expansion géographique très rapide.

Compte tenu de la grande capacité de dormance des graines, si l'arrachage et la surveillance ne sont pas effectués sur le long terme (au moins pendant une durée supérieure à la durée de dormance des graines dans le sol), l'ajonc peut rapidement se réinstaller dans ces milieux ouverts propices. Ceci explique en partie le fait que malgré une réduction importante de densité de l'ajonc, des années 1980 aux années 2010, le contour de la surface occupée reste quasi inchangé. D’ailleurs, depuis 2010, il semble que l'ajonc se soit de nouveau densifié dans certaines zones de la plaine agricole et qu'il ait recolonisé des espaces de pâturage, notamment du fait (i) de la diminution des aides financières européennes et régionales à la mécanisation (voir l'interdiction de la mécanisation dans certaines zones), (ii) du prix élevé du produit phytosanitaire employé contre l'ajonc (iii) de la démobilisation des acteurs agricoles sur la question de l'ajonc, ne transmettant pas/plus d'itinéraires techniques pour lutter contre l'ajonc et (iv) de la reprise de plusieurs exploitations par des jeunes ayant une plus faible trésorerie que les anciens. De plus, l'augmentation de la spéculation foncière, suite à une pression foncière de plus en plus forte, conduit à un maintien, si ce n'est une recrudescence, de terrains laissés à l'abandon, au moins de façon temporaire, favorisant l'expansion de l'ajonc et sa dispersion vers les parcelles les plus proches. À travers ces éléments, il apparait que la lutte contre l'ajonc sur la Plaine des Cafres est dépendante d'un ensemble de mesures prises à l'échelle familiale (main d'œuvre), locale, régionale (formation), mais aussi nationale et européenne (évolution des subventions européennes). En effet, comme l'avait déjà mentionné Chérubini (2006), les paysages agricoles réunionnais fluctuent avec l'éventail des aides octroyées dans le cadre des politiques agricoles négociées très loin des lieux d'exploitation. Ces mesures évoluant rapidement dans le temps, la dynamique de l'ajonc s'en retrouve très fluctuante d'une année sur l'autre.

76 Dans les espaces naturels, la volonté de lutte contre l'ajonc s'est mise en place au fur et à mesure de la protection légale de cette zone (Parc National de La Réunion, patrimoine mondial de l'Unesco) et sous l'effet de la catégorisation de l'ajonc comme une invasive majeure, donc comme une menace prépondérante sur la biodiversité indigène et endémique. Bien que le statut de l'espace, protégé, et celui de l'espèce, déclarée comme une des invasives majeures de l'île, concourent à une volonté d'éradication qui remonte à plus de dix ans, les moyens mis en œuvre ne permettent pas aujourd'hui d'atteindre cet objectif: les techniques de lutte mécaniques et chimiques ont une capacité de contrôle 
inférieur à la capacité de reproduction et de survie de l'ajonc, les milieux sont souvent inaccessibles, et les moyens humains et économiques sont décroissants. Il n'y a donc actuellement pas de régression de l'ajonc ; l'expansion et la densification sont toujours en cours.

L'existence d'une phase de régression est donc le fruit d'une décision de gestion assortie d'une adéquation entre les moyens mobilisables et les contraintes du terrain. Une répartition spatiale inégale des capacités de contrôle des ajoncs n'est pas propre à la Réunion. Dans leur analyse sur un large panel de zones envahies (dont l'Australie, la Nouvelle Zélande, les USA et le Chili), Hill et al. (2008) proposent la même typologie entre, d'un côté, les zones productives gérées de manière intensive où les méthodes classiques de lutte mécanique et chimiques suffisent à contenir l'ajonc et, de l'autre côté, les terres à faible rendement économique, gérées pour la préservation de la biodiversité, parfois difficiles d'accès et dans lesquelles le contrôle par ces mêmes moyens est impossible ou inefficace.

Nous avons choisi comme point d'entrée de cette étude une zone agricole et une zone naturelle, mais nous avons mis en évidence que l'ajonc ne cesse de passer les frontières entre ces deux espaces. Dès les années 1980, l'ajonc s'est dispersé des milieux agricoles de la Plaine des Cafres vers des mosaïques de milieux agricoles et naturels. Réciproquement, dans le massif naturel du Maïdo, l'expansion de l'ajonc vers les basses pentes via les ravines est en cours et des plantes ont déjà été référencées dans les zones de pâturages sous-jacentes. Cette interdépendance des milieux géographiques induit des effets de reconfiguration du milieu social en imposant, de fait, le partage entre acteurs d'une réalité commune entre ces milieux. Le contrôle de l'expansion de l'ajonc dans les milieux naturels, souhaité par les acteurs de l'environnement, ne peut être efficace sans prendre en compte les ajoncs présents en amont, dans le milieu agricole. De même, gérer les ajoncs dans les milieux agricoles juxtaposés au Massif du Maïdo, sans considérer en amont le foyer source peut vite amener à apparenter les actions de lutte au mythe de Sisyphe. Pourtant, aujourd'hui, les acteurs de ces deux types de milieu, confrontés à l'expansion de l'ajonc, n'échangent que rarement leurs informations et ne sont pas organisés en réseau. Ces observations légitiment le besoin de solidarité écologique telle que présentée par Mathevet et al. (2010) en ciblant les interdépendances entre les humains et les milieux, naturels et aménagés.

\section{Conclusion}

Les données compilées, appartenant à des champs disciplinaires distincts, ont permis en synergie d'atteindre le double objectif de cette étude, à savoir décrire le processus invasif de l'ajonc à La Réunion et comprendre l'interaction entre les facteurs humains et naturels tout au long de ce processus. Cette étude est le fruit d'une démarche foncièrement transdisciplinaire, dont les résultats dépassent le cadre des recherches spécialisées en constituant des liaisons entre disciplines à l'intérieur d'un système total sans frontières stables (Piajet 1967).

80 Nos résultats ont permis d'illustrer et de renforcer l'hypothèse de départ sur l'idée que chacune des grandes étapes du processus invasif est profondément ancrée dans l'histoire particulière des milieux et de la société humaine dans lesquels il s'opère. Pour y parvenir, plusieurs éléments et étapes de travail ont été essentiels. Le premier élément est de manier ensemble des matériaux hétéroclites, en saisissant à chaque instant de l'étude la 
moindre occasion d'enrichir le stock de données. Le second élément, qui découle logiquement du premier, est de croiser et recouper tous les différents types de données collectées par espace et par période. À ce titre, le triptyque cartographie, archives et entretiens a été très efficace dans la résolution des énigmes de départ. Il a permis parfois de confirmer, mais parfois aussi d'infirmer, certains présupposés sur les causes des introductions initiales ou des vitesses d'expansion passées et d'apporter des éléments inédits. Nous pensons que cette approche méthodologique, fruit de la transdisciplinarité, représente une réelle opportunité pour les chercheurs de tous horizons de contextualiser la dynamique des espèces invasives, apportant ainsi des éléments de compréhension des processus en jeu ainsi que des outils d'aide à la décision pour la gestion.

81 Une telle imbrication des facteurs n'est sans doute pas propre à l'ajonc et la méthode permettant de les retracer peut s'appliquer à d'autres espèces invasives, en particulier pour les plantes. Les plantes invasives ont en effet généralement été introduites volontairement, pour des raisons agricoles ou ornementales et ont souvent fait l'objet de descriptions par les botanistes, les agronomes et les géographes. De plus, ce sont souvent des espèces pionnières se développant dans des écoumènes soumis à des fluctuations d'usages et d'abandons de terres sous l'effet de l'évolution des rapports qu'entretiennent les humains avec leurs environnements. La phase de régression est également, le plus souvent, le résultat d'un choix de société entrainant la mobilisation de moyens de gestion importants, la mise en relation des différents acteurs concernés et le développement de techniques adéquates.

En conséquence, les recherches sur ce qui rend une espèce introduite envahissante et un milieu vulnérable peuvent trouver dans ce positionnement de nouvelles perspectives de réponses, à la fois à travers l'éclairage du présent par le passé et dans le rôle joué par le social, de l'échelle familiale à l'échelle globale, dans les processus invasifs.

\section{Remerciements}

Nous sommes très reconnaissants à toute l'équipe du Conservatoire Botanique National de Mascarin pour notre hébergement dans leurs locaux et pour les nombreux conseils et aides apportées sur le terrain, sur la collecte de documents historiques et la compilation cartographique. Nous remercions les agents de l'ONF et du Parc National pour les cartes de répartitions de l'ajonc et leurs conseils, ainsi que toutes les personnes qui ont accepté de s'entretenir avec nous. Nous remercions la Société Botanique de France et l'observatoire des Sciences de l'Univers de Rennes pour leur soutien financier.

\section{BIBLIOGRAPHIE}

Aikio S., Duncan R.P., \& Hulme P.E. 2010 - Herbarium records identify the role of long-distance spread in the spatial distribution of alien plants in New Zealand. Journal of Biogeography 37 : 1740-175.

Atlan A., Darrot C. 2012 - Les invasions biologiques entre écologie et sciences sociales : Quelles spécificités pour l'outre-mer français? Revue d'Écologie, Terre et Vie Sup. 11 : 101-112. 
Atlan A., Udo N., Hornoy B. \& Darrot C. 2015 - Evolution of the uses of gorse in native and invaded regions: What impacts on the dynamics and management? Revue d'Écologie, la Terre et la Vie 70, Sup. 12 « Espèces invasives » : 191-206

Barbet-Massin V., Grimaud P., Michon A. \& Thomas P. 2003 - Guide technique pour la création, la gestion et valorisation des prairies à la Réunion. Plaine des Cafres, UAFP, CIRAD, 99 p.

Beaulieu F. de \& Pouëdras L. 2014 - La mémoire des landes de Bretagne. Morlaix, Skol Vreizh, 175 p. Beltran J. 2011 - Détermination des enjeux patrimoniaux par rapport à l'envahissement de l'ajonc d'Europe et propositions de mesures conservatoires sur le massif du Piton de la Fournaise (La Réunion). Parc National de La Réunion, Université d'Avignon, 45 p.

Beltran J. \& Pausé J.-M. (Ed.) 2012 - Détermination des enjeux patrimoniaux du massif du Piton de la Fournaise (La Réunion) et propositions de mesures conservatoires par rapport à l'invasion d'une espèce exotique envahissante : l'Ajonc d'Europe (Ulex europaeus). Saint-Denis, Université de La Réunion, 29 p.

Benda P. 1956 - Recherche d'un équilibre sylvo-pastoral à La Réunion. Revue agricole de l'île de La Réunion $56-57: 141-146$.

Benoit G. 1991 - Les Hauts de La Réunion. La difficile mise en valeur d'une montagne tropicale. Bois et Forêts des Tropiques 229 : 43-50.

Bertile W. 1978 - Les Hauts de La Réunion. Étude géographique. CCUR 9 : 15-66.

Blackburn T.M., Pyšek P., Bacher S., Carlton J.T., Duncan R.P., Jarošík V., Wilson J.R.U. \& Richardson D.M. 2011 - A proposed unified framework for biological invasions. Trends in Ecology and Evolution $26: 333-339$.

Blanfort V. 1996 - Agroécologie des pâturages d'altitude à l'île de La Réunion. Pratiques d'éleveurs et durabilité des ressources herbagères dans un milieu à fortes contraintes. Paris, Université de Paris-sud, UFR Scientifique d'Orsay et CIRAD-EMVT.

Bois Joli Potier H. 1871 - Mémoire sur la colonisation de la Plaine des Cafres. La Réunion, 15 p.

Bonneuil C. \& Fressoz J.-B. 2013 - L'évènement anthropocène, la Terre, l'histoire et nous. Paris, Éditions du Seuil, $304 \mathrm{p}$.

Bonniol J.-L. \& Benoist J. 1994 - Un ordre étagé mis à bas. Contribution à une ethnologie des paysages à La Réunion. Aix-en-Provence, Laboratoire d'écologie humaine et d'anthropologie, $237 \mathrm{p}$.

Bory de Saint-Vincent J.B.G.M. 1804 - Voyage dans les quatre principales îles des mers d'Afrique. Fait par ordre du gouvernement pendant les années neuf et dix de la République (1801 et 1802). Paris, Buisson, $477 \mathrm{p}$.

Bourde de la Rogerie H. 1998 - Les bretons aux îles de France et de Bourbon (Maurice et la Réunion) aux XVIIe et XVIIIe siècles. Rennes, La Découvrance, $422 \mathrm{p}$.

Bourlet Y. 1980 - Les landes de Bretagne septentrionale. Études de biogéographie végétale. Norois 107 : 417-432.

Bréon N. 1825 - Catalogue des plantes cultivées aux jardins botanique et de naturalisation de lîle Bourbon. Saint-Denis, Imprimerie du Gouvernement, 93 p.

Brunzel S., Fischer S.F., Schneider J., Jetzkowitz J. \& Brandl R. 2009 - Neo- and archaeophytes respond more strongly than natives to socio-economic mobility and disturbance patterns along an urban-rural gradient. Journal of Biogeography 36 : 835-844. 
Butcher J.A., Collier B.A., Silvy N.J., Roberson J.A., Mason C.D. \& Peterson M.J. 2014 - Spatial and temporal patterns of range expansion of white-winged doves in the USA from 1979 to 2007. Journal of Biogeography 41 : 1947-1956.

Cadet T. 1974 - Étude de la végétation des Hautes Altitudes de l'île de La Réunion (Océan Indien). Vegetatio $29: 121-130$.

Cadet T. 1977 - La Végétation de l'île de La Réunion: étude phytoécologique et phytosociologique. AixMarseille, Université Aix-Marseille 3.

Carayol M. \& Chaudenson R. 1984 - Atlas linguistique et ethnographique de La Réunion, Tome 1. Paris, Éditions du CNRS.

CBNM (Conservatoire Botanique National de Mascarin) 2016 - Mascarine Cadetiana, système d'information géographique du Conservatoire Botanique National de Mascarin. [En ligne : http:// mascarine.cbnm.org/].

Channg Yok H. 1992 - État des lieux de la végétation de Maïdo. Saint-Denis, Université de La Réunion.

Chaudenson R. 1980 - Encyclopédie de La Réunion, tome 3, la vie rurale. Saint-Denis (La Réunion), Robert Chaudenson, $145 \mathrm{p}$.

Chauvel B., Dessaint F., Cardinal-Legrand C. \& Bretagnolle F. 2006 - The historical spread of Ambrosia artemisiifolia L. in France from herbarium records. Journal of Biogeography 33 : 665-673.

Chérubini B. 2006 - Le paysan réunionnais et les plantes envahissantes : un partenariat à construire au sein des politiques environnementales. In Auclair L., Aspe C., Beaudot P. (Ed.) Du nord au Sud: le recours à l'environnement, le retour des paysans? Aix-en-Provence, Éditions Edisud ; Paris, IRD : 199-216.

Colautti R.I., Grigorovich I.A. \& MacIsaac H.J. 2006 - Propagule Pressure: A Null Model for Biological Invasions. Biological Invasions 8 : 1023-1037.

Dalama M.-G. 2006 - L'île de la Réunion et le tourisme : d'une île de la désunion à la Réunion des Hauts et Bas. L'Espace géographique 34 : 342-349.

Defos de Rau J. 1960 - L'île de la Réunion : étude de géographie humaine. Bordeaux, Institut de géographie, Faculté des lettres, $320 \mathrm{p}$.

Delcour D. 1993 - Des hommes et un volcan : vivre à La Réunion sur le Piton de la Fournaise. Saint-Cyrles-Lecques, Delcour.

Delisle F., Lavoie C., Jean M. \& Lachance D. 2003 - Reconstructing the spread of invasive plants: taking into account biases associated with herbarium specimens. Journal of Biogeography 30 : 1033-1042.

Domènech R., Vilà M., Pino J. \& Gesti J. 2005 - Historical land-use legacy and Cortaderia selloana invasion in the Mediterranean region. Global Change Biology $11: 1054-1064$.

Dutoit T., Jäger M., Gerbaud E. \& Poschlod P. 2003 - Rôle des ovins dans le transport de graines d'espèces messicoles : le cas d'une exploitation agricole du Parc Naturel Régional du Lubéron. Courrier scientifique du Parc Naturel Régional du Luberon 7 : 68-75.

E.D. 1896 - Le Pavé. Revue agricole de l'île de La Réunion.

Facon B., Genton B.J., Shykoff J., Jarne P., Estoup A. \& David P. 2006 - A general eco-evolutionary framework for understanding bioinvasions. Trends in Ecology \& Evolution 21 : 130-135. 
Fontaine N. 1988 - La Normandie en Réunion - La colonisation de la Plaine des Palmistes et de la Plaine des Cafres de 1848 à 1872. Mémoire de maitrise d'Histoire. Saint-Denis, Université de La Réunion.

Girel J. 2006 - Quand le passé éclaire le présent : écologie et histoire du paysage. Géocarrefour 81 : 249-264.

Guellec A. 1992 - L'aménagement des Hauts à la Réunion. Annales de Géographie 101 :1-27.

Guggisberg A., Welk E., Sforza R., Horvath D.P., Anderson J.V., Foley M.E. \& Rieseberg L.H. 2012 - Invasion history of North American Canada thistle, Cirsium arvense. Journal of Biogeography 39 : 1919-1931.

Hanens Y. 1937 - L'élevage rationnel dans l'île de La Réunion. Congrès de chimie industrielle 17 : 1049-1057.

Harris C.J., Murray B.R., Hose G.C. \& Hamilton M.A. 2007 - Introduction history and invasion success in exotic vines introduced to Australia: Exotic vines in Australia. Diversity and Distributions $13: 467-475$.

Hely C. \& Forgeard F. 1998 - Hétérogénéité d'une lande haute à Ulex europaeus en relation avec la propagation du feu (Bretagne, France). Canadian journal of botany 76 : 804-817.

Hierro J.L., Maron J.L. \& Callaway R.M. 2005 - A biogeographical approach to plant invasions: the importance of studying exotics in their introduced and native range. Journal of Ecology 93 : 5-15.

Hill R.L., Ireson J., Sheppard A.W., Gourlay A.H., Norambuena H., Markin G.P., Kwong R. \& Coombs E.M. 2008 - A Global View of the Future for Biological Control of Gorse, Ulex Europaeus L. In Proceedings of the XII International Symposium on Biological Control of Weeds, La Grande Motte, France.

Holm L.G., Doll J., Holm E., Pancho J. \& Herberger J. 1997 - World Weeds: Natural Histories and Distributions. New York, NY, USA, John Wiley and Sons, Inc.

Hornoy B. 2012 - Processus évolutifs contribuant au succès envahissant de l'ajonc d'Europe, Ulex europaeus. Rennes, Université Rennes 1.

Hugues T.P. 1983 - Networks of power. Electrification in Western Society, 1880-1930. Baltimore, The Johns Hopkins University Press.

INSEE 2008 - Recensement de la population Ile de La Réunion 2008 (exploitation complémentaire). [En ligne : www.insee.fr].

Institut de recherche scientifique de Madagascar 1956 - Mémoires de l'institut scientifique de Madagascar : Entomologie. Mémoires de l'institut scientifique de Madagascar 7-8.

IGN 1957 - Carte définitive de l'île de La Réunion.

Jacob de Cordemoy C. 2004 [1862] - Itinéraire d'un voyage au volcan en 1862. In Roussin A. Album de l'île de La Réunion. Intégralité de textes et d'illustrations d'après l'édition originale en 4 volumes de 1878-1883: 570.

Jacob de Cordemoy E. 1895 - Flore de l'île de La Réunion (Phanérogames, Cryptogames vasculaires, Muscinées) avec l'indication des propriétés économiques, et industrielles des plantes. Paris, Librairie médicale et scientifique Jacques Lechevalier.

Keller S.R. \& Taylor D.R. 2008 - History, chance and adaptation during biological invasion: separating stochastic phenotypic evolution from response to selection. Ecology Letters 11 : 852-866. 
Kiss L. \& Béres I. 2006 - Anthropogenic factors behind the recent population expansion of common ragweed (Ambrosia artemisiifolia L.) in Eastern Europe: is there a correlation with political transitions? Journal of Biogeography 33 : 2156-2157.

Klepeis P., Gill N. \& Chisholm L. 2009 - Emerging amenity landscapes: invasive weeds and land subdivision in rural Australia. Land Use Policy 26 : 380-392.

Kopp A. 1937 - Économie des plantes à parfum à La Réunion. Congrès de chimie industrielle 17 : 759-762.

Kueffer C., Daehler C.C., Torres-Santana C.W., Lavergne C., Meyer J.-Y., Otto R. \& Silva L. 2010 - A global comparison of plant invasions on oceanic islands. Perspectives in Plant Ecology, Evolution and Systematics $12: 145-161$.

Larrère R. \& Larrère C. 2009 - Du « principe de naturalité » à la « gestion de la diversité biologique » In Larrère R., Lizet B., Berlan-Darqué M. (Ed.) Histoire des parcs nationaux : comment prendre soin de la nature? Versailles, Quae:205-219.

Larrère R. \& Larrère C. 2012 - Hypermodernité et sociocentrisme, In Barbier R. et al. (Ed.) Manuel de sociologie de l'environnement. Laval, Presse de l'Université Laval : 53-59.

Lavaux C. 1998 - La Réunion, du battant des lames au sommet des montagnes. Paris, Cormorans, $455 \mathrm{p}$.

Lavergne R. 1978 - Les Pestes végétales de l'île de La Réunion. Info-nature 16 : 9-60.

Lavergne R. 1980 - Les “zépinards” du Maïdo et de la Plaine des Cafres. In Lavergne R. Fleurs de Bourbon tome 2.Sainte-Clothilde (La Réunion), Imprimerie Cazal : 199-205.

Lavoie C., Jodoin Y. \& De Merlis A.G. 2007 - How did common ragweed (Ambrosia artemisiifolia L.) spread in Québec? An historical analysis using herbarium records. Journal of Biogeography 34 : 1751-1761.

Lechartier H. 2009 - Historique de l'élevage à la Plaine des Cafres et dans les Hauts du massif du Piton de la Fournaise. Lycée agricole de Saint-Paul, Parc National de La Réunion, 21 p.

Lévi-Strauss C. 1962 - La pensée sauvage. Paris, Plon, 395 p.

Lizet B. 2009 - Introduction. In Larrère R., Lizet B., Berlan-Darqué M. (Ed.) Histoire des parcs nationaux : comment prendre soin de la nature? Versailles, Quae : 11-20.

Lougnon J. 1977a - L'I.G.N. et les épinards. In Lougnon J. (Ed.), Quinze années d'actualité locales. La Réunion de 1960 à 1975. Saint-Denis, La Réunion, Imprimerie Cazal, diffusion Azalées éditions : 133-135.

Lougnon J. 1977b - Nouvelles des Hauts - Maïdo 69. In Lougnon J. (Ed.), Quinze années d'actualité locales. La Réunion de 1960 à 1975. Saint-Denis, La Réunion, Imprimerie Cazal, diffusion Azalées éditions : 305-306.

Lustenberger F. 2009 - La divagation des bovins dans les Hauts de l'ouest de La Réunion. État des lieux, analyse et propositions d'actions. Parc National de La Réunion, AgroParisTech, 59 p.

Mack R.N., Simberloff D., Lonsdale W.M., Evans H., Clout M. \& F.A. Bazzaz 2000 - Biotic invasions: causes, epidemiology, global consequences, and control. Ecological Applications 10 : 698-710.

Mahy F. de 1891 - Autour de l'île Bourbon et de Madagascar. Fragments de lettres familières. A. Lemerre, 290 p. 
Manier D.J., Aldridge C.L., O’Donnell M. \& Schell S.J. 2014 - Human Infrastructure and Invasive Plant Occurrence Across Rangelands of Southwestern Wyoming, USA. Rangeland Ecology Management 67 : 160-172.

Mathevet R., Thompson J., Delanoë O., Cheylan M., Gil-Fourrier C. \& Bonnin M. 2010 - Dossier « Le réveil du dodo III »- La solidarité écologique : un nouveau concept pour une gestion intégrée des parcs nationaux et des territoires. Natures Sciences Sociétés 18 : 424-433.

May C.P. \& Verdat M 1931 - Colonies de l'Océan indien et de l'Océan pacifique. Société d'éditions géographiques, maritimes et coloniales, $314 \mathrm{p}$.

Moreau J. 1858 - Exposition de l'agriculture, de l'industrie et des arts à La Réunion (1853). In Roussin A. Album de l'île de La Réunion. Intégralité de textes et d'illustrations d'après l'édition originale en 4 volumes de 1878-1883: 169

Moss G. 1959 - The gorse seed problem. In Proceedings of the 12th New Zealand weed control conference : 59-64.

Musée colonial de Marseille (France) 1904 - Annales du Musée colonial de Marseille.

Norambuena G.L.P. 2000 - Impact of Apion ulicis Forster on Ulex europaeus L. Seed Dispersal. Biological Control 17 : 267-271.

Odin F. \& Thuderoz C. (Ed.) 2010 - Des mondes bricolés ? : arts et sciences à l'épreuve de la notion de bricolage. Lausanne, Presses polytechniques et universitaires romandes.

Office Nationale des Forêts 1987 - Les pâturages domaniaux de la Plaine des Cafres, Bilan des actions 1978-1987. Saint-Denis, ONF.

Patu de Rosemont 1856 - Mémoire sur la colonisation des terres de l'intérieur, 21 sept. 1856. La Réunion.

Payet G. 2012 - Impact d'un incendie sur un habitat naturel en cœur de Parc National : le cas du Maïdo, île de La Réunion (Océan Indien). État des lieux et suggestions pour une gestion conservatoire à court, moyen et long terme. Parc National de La Réunion et AgroParisTech, La Réunion. 108 p.

Piajet J. 1967 - Le Système et La Classification Des Sciences. In De La Pluridisciplinarité à La Transdisciplinarité. Congrès de Locarno, 30 Avril - 2 Mai 1997, Annexes Au Document de Synthèse CIRET-UNESCO.

Pyšek P. \& Richardson D.M. 2006 - The biogeography of naturalization in alien plants. Journal of Biogeography 33 : 2040-2050.

Raunet M. 1991 - Le milieu physique et les sols de l'île de La Réunion. Conséquences pour la mise en valeur. CIRAD, Région Réunion, 515 p. [En ligne : http://open-library.cirad.fr/ files/2/522__946767618.pdf].

Rees M. \& Hill R.L. 2001 - Large scale disturbances, biological control and the dynamics of gorse populations. Journal of Applied Ecology 38 : 364-377.

Richard C. 1856 - Catalogue des végétaux cultivés au jardin du gouvernement à l'Ile de la Réunion. Typographie de LaHuppe.

Richardson R.G. \& Hill R.L. 1998 - The biology of Australian weeds. 34. Ulex europaeus L. Plant Protection Quarterly $13: 46-58$.

Rivals P. 1952 - Étude de la végétation naturelle de l'île de La Réunion. Toulouse, université de Toulouse. 
Roué M. 2009 - Construction des savoirs locaux et cogestion dans le parc national des Cévennes In Larrère R., Lizet B., Berlan-Darqué M. (Ed.) Histoire des parcs nationaux : comment prendre soin de la nature ? Versailles, Quae : 131-141.

Société nationale d'acclimatation de France 1875 - Bulletin de la Société Nationale d'acclimatation.

Société nationale d'acclimatation de France, 1925 - Revue d'histoire naturelle appliquée.

Souffrin E. 1995 - Ethno-histoire de la route du Maïdo. De la chaux aux parfums : réflexions sur la production d'un espace historique. FRAC Ile de La Réunion.

Strasberg D., Rouget M., Richardson D.M., Baret S., Dupont J. \& Cowling R.M. 2005 - An Assessment of Habitat Diversity and Transformation on La Réunion Island (Mascarene Islands, Indian Ocean) as a Basis for Identifying Broad-Scale Conservation Priorities. Biodiversity and Conservation 14 (12) : 3015-3032.

Suarez A.V. \& Tsutsui N.D. 2008 - The evolutionary consequences of biological invasions. Molecular Ecology 17 :351-360.

Tarayre M., Bowman G., Schermann-Legionnet A., Barat M. \& Atlan A. 2007 - Flowering phenology of Ulex europaeus: ecological consequences of large variability. Evolutionary Ecology 21 : 395-409.

Textor De Ravisi A.-A. 1850 - Étude sur les deux plaines des Palmistes et des Cafres de l'île de La Réunion. Saint-Denis, Typographie de Lahuppe, $106 \mathrm{p}$.

Textor De Ravisi A.-A. 1852 - Commentaires sur l'arrêté du 4 novembre 1851 sur la colonisation des deux plaines des Palmistes et des Cafres de l'île de La Réunion. Saint-Denis, Typographie de Lahuppe, $153 \mathrm{p}$.

Triolo J. \& Hoff M. 2006 - Stratégie de lutte contre l'Ajonc d'Europe (Ulex europaeus) dans les Hauts de l'Ouest. $12 \mathrm{p}$.

Université de la Réunion 1973 - Cahier du Centre universitaire de la Réunion, volume 3. Centre universitaire de la Réunion, Service des publications.

Vailland R. 1964 - La Réunion. Lausanne, Rencontre.

Vilà M. \& Ibáñez I. 2011 - Plant invasions in the landscape. Landscape Ecology 26 : 461-472.

Williams P.A. \& Karl B.J. 2002 - Birds and small mammals in kanuka (Kunzea ericoides) and gorse ( Ulex europaeus) scrub and the resulting seed rain and seedling dynamics. New Zealand Journal of Ecology 26 : 31-42.

Williams D.A., Overholt W.A., Cuda J.P., \& Hughes C.R. 2005 - Chloroplast and microsatellite DNA diversities reveal the introduction history of Brazilian peppertree (Schinus terebinthifolius) in Florida: Introduction history of Brazilian Peppertree. Molecular Ecology 14 : 3643-3656.

Wilson H.D. 1994 - Regeneration of native forest on Hinewai Reserve, Banks Peninsula. New Zealand Journal of Botany 32 : 373-383.

Wotton D.M., McAlpine K.G., 2013 - Predicting native plant succession through woody weeds in New Zealand. Wellington, New Zealand Department of Conservation, 28 p. (Doc Research and Development Series ; 336)

Zabkiewicz J.A. \& Gaskin R.E. 1978 - Effect of fire on gorse seeds. Proceedings of the 31st New Zealand Weed and Pest Control Conference : 47-52. 


\section{NOTES}

1. En sus de son métier d'agriculteur, Bois Joli Potier (de son vrai nom Henri-Laurent Potier) est également syndic de la Plaine des Cafres de 1855 à 1872. Ceci l'a conduit à effectuer le travail de maire-adjoint, de commissaire de police, de conservateur des eaux et forêts, de juge de paix et d'agent de l'administration (Fontaine 1988).

2. Ce qui correspond aux conditions climatiques qui lui sont favorables dans les régions tropicales (Hornoy 2012).

\section{RÉSUMÉS}

Les espèces invasives s'établissent souvent dans des systèmes socio-écologiques particuliers, où le rôle des humains est central. Notre étude a pour objectif d'apporter un nouveau regard sur une plante déclarée invasive majeure, l'ajonc d'Europe, Ulex europaeus, en intégrant les facteurs humains et le contexte social dans l'analyse de chaque stade du processus d'invasion. L'ajonc a été très valorisé dans sa zone d'origine, l'Europe, ce qui a induit son introduction volontaire dans de nombreuses colonies européennes vers le $\mathrm{xIX}^{\mathrm{e}}$ siècle. Il est aujourd'hui considéré comme envahissant dans plus de 30 pays et réparti sur tous les continents. Le site choisi est l'île de La Réunion; ses caractéristiques en font un laboratoire d'étude: le milieu est circonscrit, la présence humaine est récente, et les archives sont disponibles sur la quasi-intégralité de l'histoire de la colonisation. Les matériaux collectés sont composés d'extraits d'archives, de documents bibliographiques, d'entretiens semi-directifs, de cartographies, et de données biologiques. Ils ont été analysés de manière systématique, par époque et par espace. La dynamique spatio-temporelle de l'ajonc a été retracée de manière fine depuis son introduction, en liaison à la fois avec l'écologie de la plante, les caractéristiques des milieux naturels et les modifications successives des statuts politiques et économiques des espaces occupés par la plante. L'invasion biologique par l'ajonc dans l'île apparaît comme le résultat de processus complexes liant dans un «tissu sans coutures » l'interaction espèce introduite-milieu naturel et l'histoire des rapports sociaux, économiques et culturels que les humains entretiennent avec leur environnement.

Invasive species often settle in specific socio-ecological systems, in which the role of human is central. Our study aims to bring a new perspective on a plant declared as a major invasive, gorse, Ulex europaeus, incorporating human factors and the social context in the analysis of each stage of the invasion process. Gorse was highly valued in its area of origin, Europe, which has led to its voluntary introduction in many European colonies to the $19^{\text {th }}$ century. It is now considered invasive in more than 30 countries and on all continents. The site chosen is the island of Reunion, whose characteristics make it a laboratory for the study: the space is circumscribed, human presence is recent, and archives are available on almost the entire history of colonization. The collected materials are composed of archive extracts, bibliographic documents, semi-structured interviews, maps, and biological data. They were systematically analyzed by time and space. The spatiotemporal dynamics of gorse has been finely traced since its introduction, in conjunction with the ecology of the plant, the characteristics of natural environments, and successive 
changes in policies and economic status of spaces occupied by the plant. The biological invasion gorse appears as the result of complex processes binder in a seamless web interaction between introduced species and natural environment and the history of social, economic and cultural relationships that humans have with their environment.

\section{INDEX}

Mots-clés : écologie historique, processus invasif, Ulex europaeus

Index géographique : La Réunion (île de)

Keywords : Historical ecology, Ulex europaeus, Reunion Island, Invasive process

\section{AUTEURS}

\section{NATHALIE UDO}

UMR ESO, CNRS/Université Rennes 2/Agrocampus Ouest, Place du recteur Henri Le Moal. F-35043 Rennes, France

Post-doctorante

nathalie.udo88@gmail.com

\section{CATHERINE DARROT}

Maître de conférences en sociologie catherine.darrot@agrocampus-ouest.fr

UMR ESO, CNRS/Université Rennes 2/Agrocampus Ouest, Place du recteur Henri Le Moal. F-35043 Rennes, France

\section{MICHÈLE TARAYRE}

Maître de conférences en écologie michele.tarayre@univ-rennes1.fr UMR ECOBIO, CNRS/Université Rennes 1, bât. 14A, Campus de Beaulieu. F-35042 Rennes, France

\section{ANNE ATLAN}

Chargée de recherche CNRS

anne.atlan@univ-rennes2.fr

UMR ESO, CNRS/Université Rennes 2/Agrocampus Ouest, Place du recteur Henri Le Moal. F-35043 Rennes, France

UMR ECOBIO, CNRS/Université Rennes 1, bât. 14A, Campus de Beaulieu. F-35042 Rennes, France 